\title{
Robustness and Ambiguity Aversion in General Equilibrium
}

\author{
Fabio Trojani ${ }^{a, c^{*}}$ Paolo Vanini ${ }^{a, b}$ \\ ${ }^{a}$ Institute of Finance, University of Lugano, Switzerland \\ ${ }^{b}$ Zürcher Kantonalbank, Zurich, Switzerland \\ ${ }^{c}$ Faculty of Economics, University of St. Gallen, Switzerland \\ First Version: February 2000, This Version: February 2004
}

\begin{abstract}
We analyze the empirical predictions arising from settings of ambiguity aversion in intertemporal heterogenous agents economies. We study equilibria for two tractable wealth-homothetic settings of ambiguity aversion in continuous time. Such settings are motivated by a different robust control optimization problem. We show that ambiguity aversion affects optimal portfolio exposures in a way that is similar to an increase in risk aversion. A distinct property of the second of our settings of ambiguity aversion is that such increase is state-dependent and highly pronounced at moderate portfolio exposures. This feature causes quite prudent levels of equity market participation over a nontrivial set of states of the economy. In general equilibrium, ambiguity aversion tends to induce a higher equilibrium equity premium and lower interest rates. A distinct feature of the second of our settings of ambiguity aversion is that the equity premium part due to ambiguity aversion dominates when the exogenous random factors in the economy have low volatility. Thus, such setting can account for some distinct empirical predictions - like a limited equity market participation and ambiguity equity premia that dominate equity premia for small equity volatilities - which are unavailable under the first of our settings of ambiguity aversion.

Keywords: Ambiguity, Financial Equilibria, Knightian Uncertainty, Model Misspecification, Perturbation Theory, Robust Decision Making.

JEL Classification: C60, C61, G11.
\end{abstract}

\footnotetext{
${ }^{*}$ We are very grateful to the editors Marco Pagano and Joseph Zechner and two anonymous referees for very valuable comments. An earlier version of this paper appeared under the title "Risk, robustness and Knightian uncertainty in continuous time, heterogeneous agents, financial equilibria". For many valuable comments and suggestions we further wish to thank Giovanni Barone Adesi, Suleyman Basak, Michele Bernasconi, Gabrielle Demange, Larry Epstein, David Feldman, Patrick Gagliardini, Paolo Ghirardato, Thorsten Hens, Elisa Luciano, Massimo Marinacci, Paolo Porchia, Luca Rigotti, Marcel Rindisbacher, Alessandro Sbuelz, Paolo Sodini, Walter Sorana, Raman Uppal, Pietro Veronesi, conference participants in the 2001/2002 CEPR/Studienzentrum Gerzensee European Summer Symposia in Financial Markets and the 2002 EFA Annual Meeting, where this work was presented, and seminar participants at the finance seminars of the Cass Business School in London, Tilburg University, Università dell'Insubria, sede di Varese, University of Geneva, University of Lugano, University of Torino, University of Verona, University of Zurich. All remaining errors are ours. Both authors gratefully acknowledge the financial support of the Swiss National Science Foundation (grant 12-65196.01 and NCCR FINRISK). Correspondence address: Fabio Trojani, Institute of Finance, University of Lugano, Via Buffi 13, CH-6900 Lugano, e-mail: Fabio.Trojani@lu.unisi.ch.
} 


\section{Introduction}

This paper studies the empirical predictions of aversion to ambiguity (model uncertainty) in continuous-time economies with a stochastic investment opportunity set and heterogenous investors. Ambiguity refers to situations where investors do not trust in a single probability law to describe the distribution of the relevant random variables. Ambiguity aversion means that investors dislike ambiguity about the probability law of asset returns. In other words, investors prefer bets with a known probability law of future pay-offs, when compared with a situation where such a probability law is not known.

Distinguishing between ambiguity aversion and risk aversion is both economically and behaviorally important, because investors behavior under ambiguity aversion or under standard risk aversion is inherently different, as the Ellsberg (1961) paradox illustrates. Therefore, theoretical settings of ambiguity aversion should provide distinct empirical predictions with respect to set-

tings without ambiguity. We study and compare in general equilibrium such empirical predictions for a few intertemporal settings of ambiguity aversion in heterogenous agents economies. The general equilibrium analysis allows us to study the distinct effects of ambiguity aversion on the functional form of key equilibrium quantities like equity premia and interest rates. The heterogeneity structure allows us to incorporate trading and to highlight the specific implications of ambiguity aversion for equilibrium portfolio holdings.

Ambiguity aversion has been axiomatized first in an atemporal context. Gilboa (1987) proposed an axiomatic setting of ambiguity aversion where preferences can be represented by means of expected utilities with non additive probabilities. Gilboa and Schmeidler (1989) suggested a related axiomatic framework where preferences can be represented by Max-Min expected utility over a set of multiple (prior) distributions. Applications of these axiomatic settings to simple models already highlighted a few specific empirical predictions of ambiguity aversion. For instance, Dow and Werlang (1992) showed how ambiguity aversion can naturally generate limited 
equity market participation effects in investor's optimal portfolio behavior, even in the absence of transaction costs or other market frictions. Liu (2002) highlighted how such limited participation effects arise from a prudent and peculiar portfolio behavior under ambiguity aversion. Such behaviour is particularly pronounced at moderate portfolio exposures.

More recently, some authors have attempted to incorporate ambiguity aversion also in an intertemporal context. These approaches have been largely motivated or inspired by the Gilboa and Schmeidler (1989) static Max-Min expected utility setting of ambiguity aversion. ${ }^{1}$ Epstein and Wang (1994) studied some asset pricing implications of Max-Min expected utility in a discretetime infinite horizon setting. They did not, however, provide an axiomatic foundation for their model. A discrete-time axiomatic intertemporal setting of ambiguity aversion has been provided in Epstein and Schneider (2003), showing that a dynamically consistent conditional version of Gilboa and Schmeidler (1989) preferences can be represented by means of a recursive Max-Min expected utility criterion over a set of multiple (prior) distributions. Because the prevailing intertemporal utility is also recursive, they refer to it as Recursive Multiple Priors Utility (RMPU). In Chen and Epstein (2002), the RMPU setting of intertemporal ambiguity aversion was extended to continuous time. A second non axiomatic setting of intertemporal ambiguity aversion based on an alternative form of Max-Min expected utility preferences has been proposed by Hansen, Sargent and Tallarini (1999, in discrete time) and Anderson, Hansen and Sargent (AHS, 2003, in continuous time). Such a setting is based on an extension of robust control theory to an economic context.

This paper studies the empirical predictions of an aversion to ambiguity for two recent tractable settings of ambiguity aversion in continuous time. Both settings are motivated by a robust control optimization. One of them also admits a RMPU interpretation. Since investors behavior under ambiguity aversion or under standard risk aversion is inherently different, one would expect that

\footnotetext{
1 More general settings of preferences under ambiguity were proposed recently in Ghirardato et al. (2002) and Klibanoff et al. (2003), in an atemporal context. These settings permit a clearer distinction between the subjective perception of ambiguity (or, more generally, beliefs) and attitude towards ambiguity (tastes) than under Max-Min expected utility preferences. To our knowledge, no extension of these settings to an intertemporal context has been studied so far.
} 
intertemporal settings of ambiguity aversion should provide distinct empirical predictions with respect to settings without ambiguity. We analyze two tractable continuous time settings of ambiguity aversion and study which setting delivers distinct empirical predictions of ambiguity aversion, such as limited equity market participation and very prudent portfolio behaviors at moderate exposures. We first highlight the conceptual distinction between RMPU settings of ambiguity aversion and settings derived from a robust control approach. In a second step, we introduce in more detail the two specific settings of ambiguity aversion studied in the paper.

Apart from the axiomatic foundation available in RMPU theory, the conceptual distinction between the RMPU settings and the RMPU-unrelated settings is essentially one about the structure and the motivation of the set of multiple prior distributions with which expected utility is evaluated. In addition to the standard regularity conditions for set of priors in the atemporal context, RMPU yields a representation of preferences where the relevant set of priors satisfies a property called rectangularity. Rectangularity is implied by a dynamic consistency axiom imposed on conditional preferences with respect to the conditioning flow of information generated by asset prices. Rectangularity is the key feature that allows to update every prior in RMPU by means of Bayes rule and implies the strong recursive structure of the representation of preferences in RMPU. RMPU theory does not provide additional dynamic restrictions on the set of multiple priors that support its representation of preferences. Robust control theory has the goal of delivering dynamic optimal policies that are robust with respect to a slight but otherwise arbitrary misspecification of the relevant state variable dynamics. In other words, the utility performance of a robust control policy should not be too sensitive under a slight but otherwise arbitrary misspecification. $^{2}$ In AHS and related robust control settings the relevant prior distributions against

\footnotetext{
2 The naive argument that small misspecifications should not significantly affect the quantitative implications of an optimal behaviour under some particular state dynamics is generically wrong. Unfortunately, such vague statement assumes implicitly some form of "smoothness" of the performance of an optimal strategy with respect to small (local) misspecifications of the assumed state dynamics. Such smoothness cannot be granted without taking explicitly into account model misspecification in the definition and the construction of the relevant optimal policies. The importance of this point has been early recognized for instance by Huber (1981) in his influential introduction to the theory of robust statistics and has been further developed for example in econometrics to motivate several robust procedures for time series models. See Krishnakumar and Ronchetti (1997), Sakata and White (1999),
} 
which robustness is called for are described by a whole neighborhood of possible misspecifications around some fixed state dynamics. Such misspecifications are arbitrary but slight in the sense that they are constrained to have a small discrepancy from the given state dynamics. Therefore, the set of priors in the robust control approach has to deliver robustness of the prevailing optimal policies with respect to any slight misspecification of the given state dynamics. In contrast to RMPU, rectangularity - and hence the possibility of applying Bayes rule prior by prior - is not per se a crucial property of a set of multiple priors defining a robust control model. In fact, a more relevant property from a robust control perspective is the ability of a set of multiple priors to encompass a whole proximity of possible misspecifications of the assumed state dynamics. Only in such a case, a Max-Min expected utility optimization delivers optimal consumption/investment policies that are robust to any possible local misspecification of the given state dynamics. Since in the real world small misspecifications are by definition statistically hardly detectable, a useful approach is to develop robust optimal procedures that imply a satisfactory minimal utility over a whole proximity of realistic misspecifications of the assumed state dynamics. When such a robust control setting happens to be based on a rectangular set of multiple priors then it also admits a recursive representation of preferences in the form of the one supported by axiomatic RMPU theory.

This paper studies analytically the distinct asset pricing predictions of two wealth-homothetic Max-Min settings of ambiguity aversion implied by two robust control problems related to AHS's approach. The first Max-Min setting has been proposed in Maenhout (2001) who considered a homothetic version of AHS's robust control problem in order to obtain a higher analytical tractability of the arising robust Hamilton Jacobi Bellman (HJB) equations. The second MaxMin setting considered in this paper has been suggested originally in AHS (1998) and delivers a rectangular set of priors. Therefore, it can be interpreted as a continuous time version of Epstein and Schneider (2003) RMPU theory. Moreover, it implies a smooth and homothetic structure

Ronchetti and Trojani (2001), Mancini et al. (2003), Gagliardini et al. (2004) and Ortelli and Trojani (2004) for some recent work in the field. 
of the relevant robust control problems, which enhances its analytical tractability relatively to competing continuous time versions of RMPU. Chen and Epstein (2002) proposed a continuous time version of RMPU that defines constraints on the set of multiple priors which are not smooth. Therefore, such setting delivers a less tractable set of optimality conditions.

Despite our focus on homothetic settings of ambiguity aversion the class of economies analyzed in this paper includes a quite broad choice of portfolio selection and asset pricing models that were recently applied to study ambiguity aversion in intertemporal asset pricing. Some examples of applications include portfolio selection (Maenhout (2001), Lei (2001) and Trojani and Vanini (2002a), among others), option pricing with rare events (Liu et al. (2002)), models with heterogenous aversions to ambiguity (Uppal and Wang (2003)), models with time varying ambiguity (Sbuelz and Trojani (2002)) and yield curve models (Gagliardini et al. (2002)). Thus, studying equilibria in our context provides insightful characterizations of the asset pricing predictions of ambiguity aversion for a quite large class of applied models. Non homothetic robust control settings of ambiguity aversion motivated for instance by a $H_{\infty}$ or a risk sensitive control approach (see also Tornell (2000) and again AHS (2003)) do not deliver the sufficient tractability to study analytically ambiguity aversion in such generality.

The only explicit characterization of equilibrium for continuous time heterogenous economies under ambiguity aversion we are aware of is Epstein and Miao (2003). Such a paper adopts a geometric Brownian motion dynamics for the exogenous endowment process in an exchange economy where log utility investors differ only in their subjective degrees of ambiguity aversion. In this paper we study the empirical predictions of ambiguity aversion under a stochastic investment opportunity set, in complete and incomplete markets, and in the presence of heterogeneities also in risk aversions. Therefore, we provide a broader analysis of the empirical predictions of ambiguity aversion in general heterogeneous economies. Obtaining closed form solutions in the presence of a stochastic investment opportunity set is difficult and so we rely on perturbation theory to describe our equilibria. This approach allows us to study analytically the asset pricing predictions 
of ambiguity aversion and in particular to investigate the specific functional forms implied for some key equilibrium variables like equity premia and interest rates. ${ }^{3}$ To this end, we perturb the equilibria of a benchmark economy with homogeneous log utility investors with respect to the risk aversion and the ambiguity aversion parameters in our economies. This perturbation approach extends the asymptotic analysis in Kogan and Uppal (2000) from models based on standard expected utility to models including ambiguity aversion. The homothetic structure of the two settings of ambiguity aversion studied in this paper is crucial in order to develop such a perturbative analysis.

We show that for the two robust control settings studied in the paper ambiguity aversion alters optimal portfolio exposures in a way that is similar to an increase in risk aversion. Under the RMPU setting such increase is state dependent and highly pronounced at moderate portfolio exposures, causing quite prudent equity market participations over a nontrivial set of states of the economy. In general equilibrium, ambiguity aversion induces higher equilibrium equity premia and lower interest rates. Under the RMPU setting the equity premium part due to ambiguity dominates when the exogenous random factors in the economy have low volatilities. Thus, the RMPU setting accounts for some distinct asset pricing predictions of ambiguity aversion - like limited equity market participation and ambiguity equity premia that dominate equity premia for small equity volatilities - which are unavailable under the RMPU-unrelated robust control setting.

The paper is organized as follows. Section 2 introduces two general Max-Min optimization problems that model in two different ways investor's intertemporal preferences under ambiguity aversion. In Section 3, analytical solutions to the relevant partial equilibrium optimal policies are derived. These results are the necessary building blocks for the subsequent general equilibrium analysis. Section 4 develops asymptotic expressions for the relevant variables in general

\footnotetext{
3 There are basically no alternatives to perturbation methods in order to solve complicated non linear equations, as in our settings, analytically. This is particularly true for the heterogenous agents equilibria we have to consider. A non analytic in principle competing approach could use numerical procedures. Although such approach is very powerful for many problems, in our setting it is not easily applicable because of the complexity of the arising non linear equations. Moreover, by means of numerical methods alone we would get little insight into the structure and the distinct functional forms of equilibria under the different settings of ambiguity aversion studied in the paper.
} 
equilibrium, while Section 5 concludes with some summarizing remarks.

\section{The Basic Model}

This section introduces the two wealth-homothetic Max-Min robust control problems studied in the paper. Such Max-Min problems are defined by means of two different sets of multiple priors that imply different predictions of ambiguity aversion for optimal choices and asset prices. In both cases, the relevant set of multiple priors are defined by a whole proximity of slight misspecifications around a fixed state dynamics. Such fixed state dynamics is a mathematically convenient rationalization of a "fuzzy" knowledge on asset returns. We call such state dynamics for brevity the "reference model" of our investors. This terminology highlights that the reference model is an imperfect but realistic description of the true state dynamics for the economy. This viewpoint considers explicitly the fact that in real life any model is an imperfect description of the main characteristics of some observed phenomena. Therefore, models are always subject to a (possibly small) misspecification. ${ }^{4}$ Model misspecification arises when the "true" probability law of asset prices is different from the one implied by the assumed model. In our Max-Min robust control problems the relevant set of multiple prior distributions consists of a family of misspecifications of the given reference model dynamics. Such misspecifications are likely because they are constrained to be small and hardly statistically detectable. In order to constrain the relevant misspecifications to be actually small they are required to imply a moderate discrepancy relatively to the distributions under the reference model. Discrepancy is quantified by an appropriate, statistically sound, measure of diversity between distributions. We first introduce in Section 2.1 the reference model for our investors. In a second step, we outline in Section 2.2 a general class of misspecifications of the reference model that are used to define the Max-Min robust control models studied in the paper.

\footnotetext{
${ }^{4}$ For instance, the possibility of a misspecification is naturally motivated when a preferred model emerged from an empirical (formal or informal) specification analysis where alternative realistic specifications close to it where rejected.
} 


\subsection{Opportunity Set and Risk Aversions}

There are two assets, a locally risk free asset with price $B_{t}$ at time $t$ and a risky asset with price $P_{t}$ at time $t$ whose dynamics are given by

$$
\begin{aligned}
& d B_{t}=r_{t} B_{t} d t \\
& d P_{t}=\alpha_{t} P_{t} d t+\sigma_{t} P_{t}\left(\rho d Z_{t}^{X}+\sqrt{1-\rho^{2}} d Z_{t}^{P}\right)
\end{aligned}
$$

The drift and volatility $\alpha_{t}=\alpha\left(X_{t}\right)$ and $\sigma_{t}=\sigma\left(X_{t}\right)$ of the risky asset, as well as the short rate $r_{t}=r\left(X_{t}\right)$, define a stochastic investment opportunity set adapted to a state variable $X_{t}$ with dynamics

$$
d X_{t}=\zeta\left(X_{t}\right) d t+\xi\left(X_{t}\right) d Z_{t}^{X}
$$

$Z:=\left(Z_{t}^{X}, Z_{t}^{P}\right)$ is a standard two dimensional Brownian motion. The parameter $\rho$ is the correlation between the $P$ - and $X$-dynamics. For brevity, we also write $\zeta_{t}=\zeta\left(X_{t}\right), \xi_{t}=\xi\left(X_{t}\right)$.

We consider agents with time preference rate $\delta$ and power utility $u(\cdot)$ of current consumption $C_{t}$ given by

$$
u(C)=\frac{C^{\gamma}-1}{\gamma} \quad, \quad \gamma<1
$$

For $\gamma \rightarrow 0$ the $\log$ utility case follows. Agents allocate at each date $t$ fractions $w_{t}$ and $1-w_{t}$ of current individual wealth $W_{t}$ to the risky asset and the riskless asset, yielding the current wealth dynamics

$$
d W_{t}=\left[w_{t} W_{t}\left(\alpha_{t}-r_{t}\right)+r_{t} W_{t}-C_{t}\right] d t+w_{t} W_{t} \sigma_{t}\left(\rho d Z_{t}^{X}+\sqrt{1-\rho^{2}} d Z_{t}^{P}\right)
$$

\subsection{Two Settings of Intertemporal Ambiguity Aversion}

We start analyzing equilibria under ambiguity aversion and identify in the sequel model (1), (2) as the "reference model" of our investors. Let $\nu:=\left(\nu_{t}\right)_{t \geq 0}$ be a change of measure density process from the probability law under the reference model to a second probability law indexed for brevity by $\nu$. Without loss of generality we define

$$
\nu_{t}=\exp \left(\int_{0}^{t} h_{s} \cdot d Z_{s}-\frac{1}{2} \int_{0}^{t}\left|h_{s}\right|^{2} d s\right),
$$


for a suitable process $\left(h_{s}\right)=\left(h_{s}^{X}, h_{s}^{P}\right)^{\prime}$, where $\left|h_{s}\right|^{2}=h_{s}^{\prime} h_{s} . \nu$ identifies an absolutely continuous probability law that models a potential misspecification of the reference model distribution. Under a probability law $\nu$, the Girsanov Theorem gives the following for the joint $X-$ and $W$-dynamics:

$$
\begin{aligned}
d X_{t}= & {\left[\zeta_{t}+\xi_{t} h_{t}^{X}\right] d t+\xi_{t} d Z_{t}^{X}, } \\
d W_{t}= & {\left[w_{t} W_{t}\left(\alpha_{t}-r_{t}+\sigma_{t}\left(\rho h_{t}^{X}+\sqrt{1-\rho^{2}} h_{t}^{P}\right)\right)+r_{t} W_{t}-C_{t}\right] d t } \\
& +w_{t} W_{t} \sigma_{t}\left(\rho d Z_{t}^{X}+\sqrt{1-\rho^{2}} d Z_{t}^{P}\right) .
\end{aligned}
$$

If $\nu$ varies, a whole spectrum of absolutely continuous misspecifications of the reference model distribution can be obtained. Any such misspecification $\nu$ can be uniquely identified with a corresponding process $h$. The unique correspondence between $\nu$ and $h$ allows us to interpret any distributional misspecification $\nu$ as a dynamic misspecification of the reference model dynamics (1), (2). Such dynamic misspecification is implied by a corresponding process $h$ that affects the drift dynamics in (4), (5). Consequently, we can also naturally think of a misspecification $\nu$ as a dynamic drift misspecification relatively to the drift process implied by the reference model dynamics (1), (2).

We now introduce the two robust control settings of ambiguity aversion studied in the paper. They are defined by two distinct forms of intertemporal Max-Min expected utility preferences. The crucial distinction between the two settings arises because of the different sets of multiple prior distributions used to evaluate expected utilities. In both settings the set of multiple priors encompasses a whole neighborhood of slight misspecifications around the reference model probability law. This is achieved by constraining the maximal discrepancy between any misspecification's probability laws and the reference model probability distributions, where discrepancy between probability laws is quantified by means of an appropriate statistical discrepancy measure. This, in turn, amounts to constraining appropriately the size of any process $h$ associated with a misspecification $\nu$ (see again (4), (5)). Thus, in both settings investors consider a set of multiple priors consisting of small misspecifications of the given reference dynamics. Important differences 
between the sets of multiple priors studied in this paper arise because of their different intertemporal structure. Our goal is to study the distinct empirical predictions of ambiguity aversion in intertemporal Max-Min expected utility contexts based on such different sets of multiple priors.

The first setting of ambiguity aversion which we consider is characterized by a Max-Min optimization with a value function $J$ given by:

$$
(P 1) \quad: \quad J(W, X)=\left\{\begin{array}{c}
\sup _{C, w} \inf _{h} E^{h}\left[\int_{0}^{\infty} e^{-\delta t}\left(\frac{C_{t}^{\gamma}-1}{\gamma}+\frac{1}{2 \psi\left(W_{t}, X_{t}\right)} \cdot\left|h_{t}\right|^{2}\right) d t\right], \\
\text { s.t. (4), (5) }
\end{array}\right.
$$

where $\psi\left(W_{t}, X_{t}\right)>0$ is a (possibly state dependent) weighting function. Different choices of the weighting function $\psi$ have been considered in the literature. For instance, a constant weight $\psi\left(W_{t}, X_{t}\right)=\vartheta>0$ in (6) yields AHS's setting of ambiguity aversion and a robust value function given by:

$$
J(W, X)=\left\{\begin{array}{c}
\sup _{C, w} \inf _{h} E^{h}\left[\int_{0}^{\infty} e^{-\delta t}\left(\frac{C_{t}^{\gamma}-1}{\gamma}+\frac{1}{2 \vartheta} \cdot\left|h_{t}\right|^{2}\right) d t\right] \\
\text { s.t. }(4),(5)
\end{array} .\right.
$$

In AHS's setting agents select optimal policies that maximize expected utility under a worst case probability law selected from the class of admissible misspecifications $h$. The selection of the worst case probability law is constrained by the penalization factor

$$
I(\nu)=E^{h}\left[\int_{0}^{\infty} e^{-\delta t} \frac{\left|h_{t}\right|^{2}}{2} d t\right] \geq 0
$$

Such penalization constrains the size of a misspecification $\nu$ in the infimization part of $(7) . I(\nu)$ is a measure of model discrepancy between a misspecification and the reference model probability law. ${ }^{5} \quad$ Larger misspecifications $\nu$ of the reference model distribution, or equivalently larger drift misspecifications implied by a process $h$, imply a larger penalization factor (8). Because of such penalization, the worst case probability law arising from the infimization part of (7)

\footnotetext{
5 In fact, $I(\nu)$ can be shown to be a measure of the relative entropy between a misspecification $\nu$ and the reference model probability law; see for instance Hansen et al. (2001), p. 5-6. Relative entropy is a measure of distributional discrepancy that is broadly used in information theory and statistics. In statistical discrimination theory the relative entropy of two probability models can be related to the probability of a classification error in the statistical selection of one of two models based on a given amount of data. Therefore, considering misspecifications with moderate relative entropy can be interpreted as looking only at misspecifications that are statistically hardly detectable; see also AHS (2003), Section 5.
} 
cannot be arbitrarily selected: misspecifications with larger discrepancy are heavier penalized than misspecifications with small discrepancy. Furthermore, since $I(\nu)$ is weighted by $1 / \vartheta$ in $(7)$ the constraint on the selection of the worst case probability law is more stringent for low values of $\vartheta$. The extreme case arises for $\vartheta \rightarrow 0$. In this case the penalization of any misspecification $\nu$ such that $I(\nu)>0$ becomes arbitrarily large and the only worst case probability law selection implying a finite penalization is one where $I(\nu)=0$. But (8) implies that this can be the case only for a process $h$ that is identical to 0 for any time $t \geq 0$. Therefore, for $\vartheta \rightarrow 0$ the solution of (7) reduces to the one of a standard expected utility problem with no ambiguity aversion. For $\vartheta>0$, however, investors select optimal consumption/investment policies according to the probability law of a worst case misspecification selected by the first minimization in (7). The larger $\vartheta$, the larger such worst case misspecification and the set of misspecifications against which robustness has been called for. All such admissible misspecifications are characterized by a discrepancy measure $I(\nu)$ in (8) that is lower than the one of the worst case misspecification selected according to a parameter $\vartheta$. This remark highlights the specific intertemporal structure of the implicit constraints put on the set of multiple priors in the robust control optimizations (7) and (6).

AHS's choice of a constant weighting function $\psi$ in (6) implies consumption and investment policies that are typically non-homogenous functions of current wealth. In this case, (7) is typically an analytically quite intractable optimization problem. ${ }^{6}$ To avoid such intrinsic non homogeneity of AHS's robust control setting, Maenhout (2001) proposed to apply in (6) a state dependent weighting function given by

$$
\psi\left(W_{t}, X_{t}\right)=\frac{\vartheta}{\gamma J\left(W_{t}, X_{t}\right)+\frac{1}{\delta}} \quad, \quad \vartheta>0 \quad .
$$

Hence, in this setting the penalization for the discrepancy of a misspecification depends on the indirect utility continuation value $J$ itself. Such choice of $\psi$ implies consumption/investment robust optimal policies that are homogenous functions of current wealth and robust HJB equations

\footnotetext{
${ }^{6}$ Infinite order asymptotic solutions for AHS's robust control problem under a constant investment opportunity set have been obtained by Trojani and Vanini (2001).
} 
that are analytically tractable in some economically interesting dynamic settings. This paper makes use of problem (P1) with the specification (9) as a first setting of ambiguity aversion and studies its general equilibrium empirical predictions.

The second setting of ambiguity aversion we address is based on a robust control problem with a value function given by:

$$
(P 2) \quad: \quad J(W, X)=\left\{\begin{array}{c}
\sup _{C, w} \inf _{h} E^{h}\left[\int_{0}^{\infty} e^{-\delta t} \frac{C_{t}^{\gamma}-1}{\gamma} d t\right] \\
\text { s.t. (4), (5) and } \frac{1}{2} h^{\prime} h \leq \eta
\end{array} .\right.
$$

As in problem (P1), the optimization (10) is a Max-Min expected utility optimization over a constrained set of admissible misspecifications $\nu$. Such set is defined by a constraint on the squared norm of any process $h$ defining an admissible drift misspecification (4), (5). The constraint $\frac{1}{2} h^{\prime} h \leq \eta$ in (10) implies that for any time $t \geq 0$ the squared Euclidean norm $h_{t}^{\prime} h_{t}$ of the process $h$ at that time is bounded by $2 \eta$. More specifically, the set of admissible misspecifications in the constrained optimization (10) can be written as

$$
\left\{h \quad: \quad h_{t}^{\prime} h_{t} \in[0,2 \eta] \text { for any } t \geq 0\right\}
$$

and defines a rectangular set of priors, in Chen and Epstein (2002, p. 8) terminology. ${ }^{7}$ Consequently, the preferences implied by problem (P2) are a continuous time version of axiomatic RMPU theory. The key property of rectangular sets of multiple priors is that they can be constructed by arbitrary selections from primitive sets of transition densities between $t$ and $t+d t$, $t \geq 0$. The set (11) is rectangular because any process $h$ (and hence any density process $\nu$ ) in (11) corresponds to a selection of transition densities from $t$ to $t+d t, t \geq 0$, such that $h_{t}^{\prime} h_{t} \in[0,2 \eta] .{ }^{8}$

\footnotetext{
7 It can be shown that such constraint is equivalent to imposing for any $t \geq 0$ a bound $\eta$ on the statistical discrepancy between all transition probabilities from $t$ to $t+d t$ under an admissible misspecification and those under the reference model. More precisely, such constraint limits the growth rate of the relative entropy of a misspecification over time. Indeed:$$
E^{h}\left[\int_{t+\Delta t}^{\infty}{\frac{\left|h_{s}\right|^{2}}{2}}^{2} d s\right]-E^{h}\left[\int_{t}^{\infty} \frac{\left|h_{s}\right|^{2}}{2} d s\right]=E^{h}\left[\int_{t+\Delta t}^{\infty} \ln \left(\nu_{s}\right) d s\right]-E^{h}\left[\int_{t}^{\infty} \ln \left(\nu_{s}\right) d s\right]
$$

Since the RHS of this equation is the variation in the relative entropy of a misspecification over the period $t, t+\Delta t$, the claim follows. See also AHS (1998) and Hansen et al. (2001) for more details.

8 The distinction from Chen and Epstein (2002)" $k$-ignorance" continuous time version of RMPU arises by the
} 
For $\eta \rightarrow 0$, problem (P2) coincides with a standard expected utility maximization. For $\eta>$ 0 agents select optimal policies that maximize expected utility under a worst case probability law selected from the class (11) of admissible misspecifications. This is a Max-Min pessimistic optimization that depicts ambiguity aversion. The larger $\eta$, the larger is the set (11) of relevant misspecifications in problem (P2) and the more ambiguity averse agents preferences are.

The set of multiple prior distributions implicit in the penalized formulation of a robust optimization of the form (6) is not as easily identifiable as the set (11). To highlight a more direct link with Max-Min expected utility theory, Hansen et al. (2001) showed by means of the Lagrange

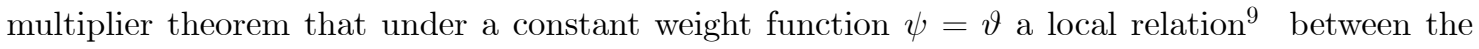
preferences behind the penalized problem (6) and those of a constrained Max-Min expected utility optimization can be established. In such a constrained optimization, the relevant constrained set of multiple (prior) distributions is given as:

$$
\left\{h: E^{h}\left[\int_{0}^{\infty} e^{-\delta t} \frac{\left|h_{t}\right|^{2}}{2} d t\right] \leq k(\vartheta)\right\}
$$

for some positive constant $k(\vartheta)$ depending on $\vartheta$. Such a set of multiple priors is clearly not rectangular. ${ }^{10}$ Therefore, the implied constrained Max-Min expected utility optimization does not admit a RMPU representation, in contrast to the optimization problem (10).

To further highlight the structural differences between the penalized problem (6), (9) and the constrained problem (10), one can apply the Lagrange multiplier theorem in order to relate locally the preferences of problem (10) to those of an associated penalized problem (6) with a corresponding stochastic weight function $\psi$. In fact, when comparing the worst case model specific way of defining such rectangular sets of multiple priors. In our context, a rectangular set of priors defined by a " $k$-ignorance" metric would be:

$$
\left\{h:\left|h_{t}^{P}\right| \leq k_{P},\left|h_{t}^{X}\right| \leq k_{X} \text { for any } t \geq 0\right\}
$$

for some non negative constants $k_{X}, k_{P}$. Such metric constrains in a nonsmooth way every component of the process $h$. This is the reason why it implies a less tractable intertemporal optimization problem under ambiguity aversion than problem (P2).

9 Such relation is local in the sense that it says nothing about preferences orderings off the optimal solution path.

10 A simple proof is given for completeness in the Appendix. 
solutions under the setting (P1) and (P2) - which are given in equation (56) and (61) of the Appendix, respectively - it appears that the weighting function in a penalized form of problem (10) has to be:

$$
\psi\left(W_{t}, X_{t}\right)=\frac{\sqrt{2 \eta}}{\left(W^{2} J_{W}^{2} \sigma^{2}+\xi^{2} J_{X}^{2}+2 \sigma_{X P} W J_{X} J_{W}\right)^{\frac{1}{2}}},
$$

and depends on the square root of the quadratic variation in the prevailing continuation utility, as opposed to the level of continuation utility that is present in (9). The weight function $\psi$ in (13) will be therefore particularly high when the quadratic variation in continuation utilities is low. The weight function (9), instead, will be particularly high when continuation utilities are low. These are structurally huge differences between the optimization (6), (9) and problem (10). Moreover, problem (10) admits a simple direct interpretation as axiomatic RMPU theory, as we have discussed above.

\section{Optimal Consumption and Investment Policies}

This section studies the optimal consumption-investment policies to problems (6) and (10). We provide asymptotic solutions that characterize analytically the optimal behavior arising under such settings of ambiguity aversion. These results are necessary for the general equilibrium analysis in Section 4. The basic idea of asymptotic methods is to formulate a general problem, find a particular relevant case that has a known solution, and use this as a starting point for computing the solution to nearby problems. Since in our general equilibrium analysis we will focus for tractability on perturbations of a representative agent log utility economy, in this section perturbations are performed around an expected log utility investor's problem. Perturbations are with respect to two parameters: the risk aversion parameter $\gamma$ and the ambiguity aversion parameters $(\vartheta$ or $\eta)$. Hence, our asymptotic solutions hold for neighborhoods of a model with log utility of consumption and no ambiguity aversion. The homotheticity of the robust control problems (6) and (10) allows us to extend in a quite natural way the analysis in Kogan and Uppal (2000) from economies with 
no ambiguity to settings of ambiguity aversion. ${ }^{11}$

\subsection{Ambiguity Aversion: Problem (P1)}

The homogeneity of the Max-Min problem (6) under the specification (9) implies a value function $J$ given by

$$
J(W, X)=\frac{1}{\delta} \cdot \frac{\left(e^{g(X)} W\right)^{\gamma}-1}{\gamma}
$$

for some unknown function $g(X)$. Since in general the function $g$ cannot be computed in closed form, the key idea is to approximate it. To this end, we expand $g$ to first order in $(\gamma, \vartheta)::^{12}$

$$
g=g_{0}+\gamma g_{1}+\vartheta g_{2}+O^{2}(\gamma, \vartheta)
$$

By construction, $g_{0}$ solves $(6)$ for $\gamma, \vartheta \rightarrow 0$, i.e. the expected utility problem of an investor with logarithmic utility of consumption . Writing $J_{\log }(X, W)$ for this solution, (14) implies for $\gamma, \vartheta \rightarrow 0$ :

$$
J_{\log }(X, W)=\frac{1}{\delta}\left(\ln (W)+g_{0}(X)\right)
$$

Hence, $g_{0}$ is given in closed form for problems were $J_{\log }$ can be computed explicitly. It turns out that $g_{0}$ is all what we need to approximate the optimal policies to problem (6) up to first order in $(\gamma, \vartheta)$. This is the next proposition.

Proposition 1 The first order asymptotics for the optimal policies of problem (6) under the specification (9) are

$$
\begin{gathered}
c(X)=\delta\left(1-\gamma\left(g_{0}(X)-\ln (\delta)\right)\right)+O^{2}(\gamma, \vartheta) \\
w(X)=\frac{1}{1-(\gamma-\vartheta)}\left(\frac{\alpha-r}{\sigma^{2}}+(\gamma-\vartheta) \frac{\partial g_{0}(X)}{\partial X} \cdot \frac{\sigma_{X P}}{\sigma^{2}}\right)+O^{2}(\gamma, \vartheta)
\end{gathered}
$$

where $\sigma_{X P}=\rho \xi \sigma$ and $c=C / W$ is the optimal consumption to wealth ratio.

We observe from Proposition 1 that optimal consumption is not altered by ambiguity aversion because the parameter $\vartheta$ is absent in the consumption policy (16). Thus, the impact of ambiguity aversion on optimal consumption is of higher order in $(\gamma, \vartheta)$. On the contrary, optimal investment in (17) is affected by ambiguity aversion. The first order consumption policy (16) can be

\footnotetext{
11 The analysis of non-homothetic robust control settings by means of asymptotic methods is more difficult; see for instance Trojani and Vanini (2001) and Trojani and Vanini (2002b).

12 Hereafter $O^{2}(\gamma, \vartheta)$ is the Landau symbol that we use to denote terms of second order in $(\gamma, \vartheta)$.
} 
interpreted as the optimal consumption policy of an expected utility investor with elasticity of intertemporal substitution $1 /(1-\gamma)$. The first order investment policy (17) can be interpreted as the optimal investment policy of an expected utility investor with relative risk aversion $1-(\gamma-\vartheta)$. Hence, to first order ambiguity aversion in problem (6), (9) yields optimal policies that are observationally equivalent to those of a setting with an increased "effective" risk aversion parameter $1-(\gamma-\vartheta)$ and an elasticity of intertemporal substitution $1 /(1-\gamma)$.

The fact that the functional form of the optimal investment policy (17) is the same as the one of an expected utility model with a suitable risk aversion parameter implies an investment behavior that is observationally equivalent to one without ambiguity. To study the impact of ambiguity aversion on optimal investment we split the optimal policy (17) in a myopic and a hedging part:

$$
w=\underbrace{\frac{1}{1-(\gamma-\vartheta)} \cdot \frac{\alpha-r}{\sigma^{2}}}_{\text {Myopic demand }}+\underbrace{\frac{\gamma-\vartheta}{1-(\gamma-\vartheta)} \frac{\partial g_{0}}{\partial X} \cdot \frac{\sigma_{X P}}{\sigma^{2}}}_{\text {Hedging demand }}+O^{2}(\gamma, \vartheta)
$$

To be more specific, we write the myopic demand $w^{M}(\vartheta)$ and the demand for intertemporal hedging $w^{H}(\vartheta)$ as

$$
w^{M}(\vartheta)=\frac{\phi}{\sigma(1-\gamma)} \cdot \frac{1-\gamma}{1-(\gamma-\vartheta)}=w^{M}(0) \frac{1-\gamma}{1-(\gamma-\vartheta)}
$$

and

$$
w^{H}(\vartheta)=\frac{\rho \psi \gamma}{\sigma(1-\gamma)} \cdot \frac{1-\gamma}{1-(\gamma-\vartheta)} \cdot \frac{\gamma-\vartheta}{\gamma}=w^{H}(0) \underbrace{\frac{1-\gamma}{1-(\gamma-\vartheta)}}_{A} \cdot \underbrace{\frac{\gamma-\vartheta}{\gamma}}_{B} .
$$

It follows from (19) that ambiguity aversion always reduces myopic risky exposures. Intuitively, this is due to the increased effective risk aversion parameter $1-(\gamma-\vartheta)$. The relative portfolio $w^{M}(\vartheta) / w^{M}(0)$ is precisely the ratio of the effective risk aversion parameters $1-\gamma$ and $1-(\gamma-\vartheta)$ when $\vartheta=0$ (no ambiguity) and when $\vartheta>0$ (ambiguity), respectively. From (20) the intertemporal hedging relative portfolio $w^{H}(\vartheta) / w^{H}(0)$ is the product of two terms $A$ and $B$. The term $A$ derives again from an increase in effective risk aversion, which tends to decrease the absolute demand for hedging when $\vartheta>0$. The term $B$ arises because under the worst case probability law selected in (6) the relevant $X$ - dynamics is different from the reference model dynamics (1), (2). Hence, 
optimal intertemporal hedging is modified accordingly, in order to hedge intertemporal changes in worst case opportunity sets and the associated marginal utilities.

Finally, we remark that in the present setting of ambiguity aversion the optimal policies $w^{M}(\vartheta)$ and $w^{H}(\vartheta)$ are linear functions of $\phi=\frac{\alpha-r}{\sigma}$ and $\psi=\xi \frac{\partial g_{0}}{\partial X}$. The same holds for the optimal policies $w^{M}(0)$ and $w^{H}(0)$ in the absence of ambiguity. Since in the absence of ambiguity aversion optimal portfolios are well known to reflect risk aversion with respect to second order in volatility risk, we conclude that the present setting of ambiguity aversion implies this same type of portfolio behaviour.

\subsection{Ambiguity Aversion: Problem (P2)}

The homogeneity of the Max-Min problem (10) implies again a value function $J$ of the form

$$
J(W, X)=\frac{1}{\delta} \cdot \frac{\left(e^{g(X)} W\right)^{\gamma}-1}{\gamma}
$$

for some unknown function $g(X)$. We expand $g$ in $(\gamma, \sqrt{2 \eta})$ to first order: ${ }^{13}$

$$
g=g_{0}+\gamma g_{1}+\sqrt{2 \eta} g_{2}+O^{2}(\gamma, \sqrt{\eta})
$$

By construction, $g_{0}$ solves (10) for $\gamma, \eta \rightarrow 0$. Therefore, the zeroth order solution to problem (10) is again the value function $J_{\log }(X, W)$ in (15). Although (6) and (10) are very different optimization problems, it turns out that also in the present setting the first order optimal policies under ambiguity aversion can be fully characterized by means of only the zeroth order function $g_{0}$

Proposition 2 The asymptotic expansions for the optimal policies of problem (10) are

$$
\begin{aligned}
c(X) & =\delta\left(1-\gamma\left(g_{0}(X)-\ln (\delta)\right)\right)+O^{2}(\gamma, \sqrt{\eta}), \\
w(X) & =\frac{1}{1-\left(\gamma-\sqrt{\frac{2 \eta}{G_{0}(X)}}\right)}\left(\frac{\alpha-r}{\sigma^{2}}+\left(\gamma-\sqrt{\frac{2 \eta}{G_{0}(X)}}\right) \frac{\partial g_{0}}{\partial X} \frac{\sigma_{X P}}{\sigma^{2}}\right)
\end{aligned}
$$

where

$$
G_{0}(X)=\left(\frac{\alpha-r}{\sigma}\right)^{2}+\left(\xi \frac{\partial g_{0}}{\partial X}\right)^{2}+2\left(\frac{\alpha-r}{\sigma}\right) \rho \xi \frac{\partial g_{0}}{\partial X}
$$

$\sigma_{X P}=\rho \xi \sigma$ and $c=C / W$ is the optimal consumption to wealth ratio.

${ }^{13}$ Hereafter $O^{2}(\gamma, \sqrt{\eta})$ is a symbol that we use to denote terms of second order in $(\gamma, \sqrt{\eta})$. 
Just as for the ambiguity setting of the last section, first order optimal consumption (22) is not affected by ambiguity aversion. In fact, the consumption asymptotics (16) and (22) are identical. Ambiguity aversion influences the optimal investment policy (23), which can be reinterpreted as the optimal portfolio strategy of an investor with an effective risk aversion $1-\left(\gamma-\sqrt{2 \eta / G_{0}}\right)$. In contrast to the previous setting, such effective risk aversion is state dependent via the function $G_{0}(X) . G_{0}(X)$ depends on the state $X$ only through a positive definite form in $\phi=\frac{\alpha-r}{\sigma}$ and $\psi=\xi \frac{\partial g_{0}}{\partial X}$. The myopic and hedging portfolios $w^{M}(\eta)$ and $w^{H}(\eta)$ under ambiguity aversion can be now written as

$$
w^{M}(\eta)=w^{M}(0) \frac{1-\gamma}{1-\left(\gamma-\sqrt{\frac{2 \eta}{G_{0}}}\right)}
$$

and

$$
w^{H}(\eta)=w^{H}(0) \frac{1-\gamma}{1-\left(\gamma-\sqrt{\frac{2 \eta}{G_{0}}}\right)} \cdot \frac{\gamma-\sqrt{\frac{2 \eta}{G_{0}}}}{\gamma} .
$$

For any given state level $X$, the structure of the portfolio components (24) and (25) is similar to the one in (19) and (20). However, when we consider the portfolio functional forms (24) and (25) in dependence of the underlying state $X$ we remark that the relative portfolios $w^{M}(\eta) / w^{M}(0)$ and $w^{H}(\eta) / w^{H}(0)$ are now state dependent functions of $G_{0}(X)$. That is, ambiguity aversion now affects optimal portfolios in a non uniform way over the relevant support of $X$. This is a striking difference with the setting of ambiguity aversion discussed in the last section.

The relative portfolios $w^{M}(\eta) / w^{M}(0)$ and $w^{H}(\eta) / w^{H}(0)$ are now nonlinear functions of $\phi$ and $\psi$. The smallest relative portfolios arise for $G_{0}(X) \rightarrow \infty$, i.e. for $\phi \rightarrow \pm \infty$ or $\pm \psi \rightarrow \infty$. In this case $w^{M}(\eta) / w^{M}(0)$ and $w^{H}(\eta) / w^{H}(0)$ converge to 1 . For $G_{0}(X) \rightarrow 0$, i.e. for $\phi \rightarrow 0$ and $\psi \rightarrow 0, w^{M}(\eta) / w^{M}(0)$ converges to 0 while $w^{H}(\eta) / w^{H}(0)$ converges to $(1-\gamma) / \gamma$. Hence, the largest relative portfolios now arise precisely when $\phi, \psi \rightarrow 0$, i.e. when portfolio exposures in the absence of ambiguity aversion are small. This is a striking difference with the last section where such relative portfolios were constant as functions of the state $X$. Thus, the current setting of ambiguity aversion induces some very distinct predictions on optimal portfolio behaviour. 
An interesting special case of Proposition 2 arises in the perfect correlation case $\rho= \pm 1$. In this case, $G_{0}$ is a perfect square and $\sqrt{G_{0}}$ collapses to an absolute value expression. This gives the next result.

Proposition 3 Assume a perfect correlation setting such that $\rho= \pm 1$ and let

$$
w^{ \pm}(X)=\frac{1}{\sigma^{2}(1-\gamma)}\left(\alpha-r+\gamma \frac{\partial g_{0}}{\partial X} \xi \sigma \pm \sqrt{2 \eta} \sigma\right) .
$$

The asymptotics of the optimal investment policy $w(X)$ are:

1. If $w^{-}(X)>-\frac{\xi}{\sigma} \frac{\partial g_{0}}{\partial X}(X)$ :

$$
w(X)=w^{-}(X)+O^{2}(\gamma, \sqrt{\eta})
$$

2. If $w^{+}(X)<-\frac{\xi}{\sigma} \frac{\partial g_{0}}{\partial X}(X)$

$$
w(X)=w^{+}(X)+O^{2}(\gamma, \sqrt{\eta})
$$

3. If neither 1. nor 2. are satisfied

$$
w(X)=-\frac{\xi}{\sigma} \frac{\partial g_{0}}{\partial X}(X)+O^{2}(\gamma, \sqrt{\eta}) \quad .
$$

We can reinterpret the optimal investment rules $w^{-}(X)$ and $w^{+}(X)$ under 1 . and 2. as the optimal portfolios of a precautionary investor who modifies excess returns $\alpha-r$ by a correction factor $\mp \sqrt{2 \eta} \sigma$. Such correction factor penalizes the ambiguity about the underlying reference model for asset prices. The third type of portfolio profile arises in states of the world where investors are able to avoid completely the conditional ambiguity about the underlying reference model dynamics. It arises if and only if $w^{-} \leq-\frac{\xi}{\sigma} \partial g_{0} / \partial X$ and $w^{+} \geq-\frac{\xi}{\sigma} \partial g_{0} / \partial X$. Under the portfolio profiles $w^{-}(X)$ and $w^{+}(X)$ in 1 . and 2. investors are exposed to ambiguity and the selected worst case misspecifications are non zero. This is taken into account by correcting excess returns $\alpha-r$ by the correction factor $\pm \sqrt{2 \eta} \sigma$. Such adjustment yields portfolios with a performances that is less exposed to a misspecification of the reference model dynamics for excess equity returns. For states where investors are able to fully avoid ambiguity, the optimal portfolios imply a zero conditional model discrepancy between the reference model and any misspecification. The conditional impact of a reference model misspecification on the resulting continuation utility is then completely absent. This is possible because the underlying wealth and state dynamics are 
perfectly correlated. In particular, we remark that under the portfolio profile 3 . in Proposition 3 investors will typically have a zero myopic exposure to equity. Moreover, as soon as intertemporal hedging motives are moderate for such states of the economy, i.e. $\partial g_{0} / \partial X \approx 0$, equity portfolio exposures will be virtually zero and investors will avoid investing in the equity market. In general equilibrium, such features will imply some very prudent equity market participations under the present setting of ambiguity aversion.

Finally, it is important to remark that also in the above case $\rho= \pm 1$ the portfolio policies implied by problem (P2) and (P1) in Proposition 3 and 1, respectively, are economically and behaviorally different. First, in Proposition 3 the optimal portfolio policy has a different functional form for different values of the underlying state variable, while in Proposition 1 one single functional form holds for the optimal policy over the whole relevant support of $X$. Second, even if we focus exclusively one the portfolio profiles 1 . and 2. in Proposition 3, differences with the policy under problem (P1) do generally arise. To see this more explicitly consider for brevity the profile 2. in Proposition 3:

$$
w^{+}(X)=\frac{1}{\sigma^{2}(1-\gamma)}\left(\alpha-r+\gamma \frac{\partial g_{0}}{\partial X} \xi \sigma-\sqrt{2 \eta} \sigma\right),
$$

and compare it to the optimal policy for problem (P1) implied by Proposition 1:

$$
w(X)=\frac{1}{\sigma^{2}(1-\gamma+\vartheta)}\left(\alpha-r+(\gamma-\vartheta) \frac{\partial g_{0}}{\partial X} \xi \sigma\right) .
$$

These two optimal policies can be of the same functional form only in the very specific case that

$\frac{\partial g_{0}}{\partial X} \xi$ is a constant. Such a case arises for instance when the opportunity set is not stochastic; see also Maenhout (2001), Trojani and Vanini (2002) and Uppal and Wang (2003).

\subsection{Some Explicit Partial Equilibrium Computations}

A more explicit discussion of the formulas derived in the last sections can be developed for models where $g_{0}$ can be computed explicitly. We illustrate the above findings for a version of Kim and 
Omberg's (1996) model with dynamics given by:

$$
\begin{aligned}
d B_{t} & =r B_{t} d t, \\
d P_{t} & =\alpha_{t} P_{t} d t+\sigma P_{t} d Z_{t}^{P}, \\
d X_{t} & =\lambda\left(\bar{X}-X_{t}\right) d t+\xi d Z_{t}^{X},
\end{aligned}
$$

where $r, \sigma, \xi, \lambda, \bar{X}>0$, and $\alpha_{t}=r+\sigma X_{t}$. In this setting $X$ represents the reference model market price of risk on equity. The function $g_{0}$ is a quadratic polynomial $a_{0}+a_{1} X+\frac{1}{2} a_{2} X^{2}$, with coefficients $a_{0}, a_{1}, a_{2}$ defined in the footnote. ${ }^{14}$ This implies two perfectly correlated processes $\phi=\frac{\alpha-r}{\sigma}$ and $\psi=\xi \frac{\partial g_{0}}{\partial X}$ which are both linear functions of $X$. The function $G_{0}(X)$ is a nonnegative second order polynomial of $X$. Therefore, to first order optimal portfolios under the setting (P1) of ambiguity aversion are a linear function of $X$, precisely as in the setting with no ambiguity aversion $(\vartheta=0)$; see again (17). Optimal portfolios under the setting (P2) of ambiguity aversion are non linear functions of $X$. Moreover, since $G_{0} \rightarrow 0\left(G_{0} \rightarrow \infty\right)$ if and only if $X \rightarrow 0$ $(X \rightarrow \pm \infty)$ the largest (smallest) relative portfolio effects under ambiguity aversion arise for moderate (extreme) absolute values of the reference model risk premium $\alpha_{t}-r$; see again (23).

Figure 1 illustrates such differences in optimal portfolio behavior for some choices of the model parameters.

\section{Insert Figure 1 about here}

The left panel of Figure 1 compares the portfolio policies in the absence of ambiguity aversion (the black straight line) with those under the settings (P1) (the dash dotted and dashed straight lines) of ambiguity aversion. The classical policy in the absence of ambiguity aversion is an increasing

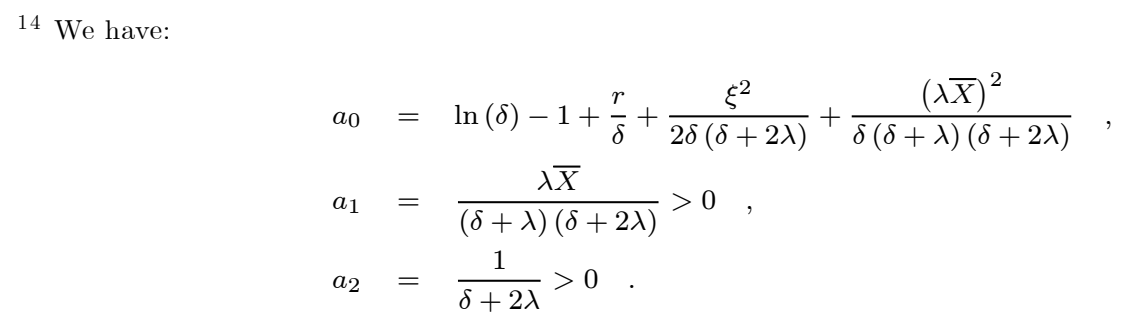

A proof is given in the Appendix for completeness. 
straight line as a function of the reference model market price of risk $X$. The policies under the setting (P1) of ambiguity aversion have the same functional form but their slope is smaller. The larger $\vartheta$, the smaller the slope. The policies under the setting (P2) of ambiguity aversion are presented in the right panel of Figure 1. They have a different functional form and present an $S$-shaped behavior around the point $X=0$. In this region, portfolio exposures are about zero. For instance, for values of $X$ in the interval $[-0.05,0.05]$ they are between \pm 0.05 . Over such an interval of $X$ values, the classical portfolio policy and the one under the setting (P1) of ambiguity aversion already imply substantial portfolio exposures. To emphasize further this point, Figure 2 plots the differences between the portfolio policies in the setting (P1) and those in the setting (P2) of ambiguity aversion for the same parameter choices as in Figure 1.

\section{Insert Figure 2 about here}

\section{General Equilibrium}

We consider heterogenous agents general equilibria. More specifically, we study economies where there are two agents with utilities of current consumption given by

$$
u(C)=\frac{C^{\gamma}-1}{\gamma} \quad, \quad u\left(C^{(1)}\right)=\log \left(C^{(1)}\right) \quad, \quad \gamma<1
$$

and ambiguity aversion parameters given by

$$
\vartheta>0 \quad, \quad \vartheta^{(1)}=0 \quad\left(\eta>0 \quad, \quad \eta^{(1)}=0\right)
$$

We assume the same time preference rate $\delta$ for all agents. In the sequel, we start by an example of a complete production economy and analyze in a second step an example of an incomplete exchange economy. Such economies have been studied in Kogan and Uppal (2000) for settings with no ambiguity aversion. For the production economy we focus on ambiguity aversion in the setting of problem (P2), because it is the setting that delivers the most interesting distinct empirical predictions of ambiguity aversion, namely an endogenous dynamic equity market participation 
in the absence of transaction costs or other market frictions. Such participation is endogenous because it depends on the degree of ambiguity aversion and on the prevailing cross-sectional wealth distribution in the economy. In the production economy example, we explicitly characterize conditions (see (35) below) under which limited equity market participation arises in equilibrium. For the exchange economy, we discuss in a unified framework the predictions under the settings (P1) and (P2) by focusing on the distinct equilibrium portfolio behaviors arising under ambiguity aversion and the different predictions for the functional form of equity premia.

\subsection{Production Economies}

We consider an extended version of the production economy in Dumas (1989) where a single constant returns to scale technology with dynamics

$$
d S_{t}=\left(\alpha S_{t}-C_{t}-C_{t}^{(1)}\right) d t+\sigma S_{t} d Z_{t}
$$

for the aggregate capital stock, with $\alpha, \sigma>0$, is assumed. The risky asset is a stock on the production technology with cumulative return process

$$
d P_{t}=\alpha P_{t} d t+\sigma P_{t} d Z_{t}
$$

The number of shares in this economy is equal to the aggregate capital stock. The riskless asset is a money account with price dynamics

$$
d B_{t}=r_{t} B_{t} d t
$$

where $r_{t}$ is an interest rate that has to be determined in equilibrium. In such a setting markets are complete since a single Brownian motion affects the production and stock price dynamics.

Definition 4 We call a process $\left(S_{t}, r_{t}, w_{t}, w_{t}^{(1)}, c_{t}, c_{t}^{(1)}\right)$ an asymptotic equilibrium under ambiguity aversion if:

- The individual portfolio and consumption rules $w_{t}, w_{t}^{(1)}, c_{t}, c_{t}^{(1)}$ are asymptotically optimal, i.e. they satisfy the consumption rule (22) and the investment rules in Proposition 3.

- The financial markets clear: $w_{t} W_{t}+w_{t}^{(1)} W_{t}^{(1)}=W_{t}+W_{t}^{(1)}$. 
In general equilibrium the relevant state variable is the cross-sectional wealth distribution $\omega_{t}$ in the economy: $X_{t}=\omega_{t}:=W_{t} /\left(W_{t}+W_{t}^{(1)}\right)$. Furthermore, the function $g_{0}$ in Propositions 1,2 and 3 is now endogenous to the economy, i.e. it depends on $\gamma$ and $\eta$. However, it can be expanded in a neighborhood of the representative agent value function of an homogeneous economy with log utility investors $\left(\gamma=\gamma^{(1)}=\eta=\eta^{(1)}=0\right)$. Denoting this value function by

$$
J_{\log , 0}(X, W)=\frac{1}{\delta}\left(\log (W)+g_{00}(X)\right),
$$

it turns out that to characterize the desired rules completely we only need to determine $g_{00}$ and $r$ as a function of $g_{00}$. Moreover, by definition $g_{00}$ is completely determined by the representative-agent $\log$ utility solution and it is a constant. For this benchmark economy, market clearing implies that $\alpha-\sigma^{2}$ is the constant equilibrium interest rate. Using formula (41) in Kogan and Uppal (2000), $g_{00}$ is given by

$$
g_{00}=\log (\delta)-1+\frac{\alpha-\sigma^{2} / 2}{\delta}
$$

To characterize the equilibrium under the setting (P2) of ambiguity aversion, we need to decompose the state space of $\omega_{t}$. Such a decomposition is necessary because of the different portfolio profiles for the complete markets case in Proposition 3. In general equilibrium only two portfolio profiles can arise. They correspond to the portfolio policies (26) and (28) in Proposition 3. Moreover, since the equilibrium function $g_{00}$ in (34) is constant, the equilibrium portfolio policy (28) coincides to first order with a zero investment strategy in the risky asset. To characterize the states of the economy where the two different portfolio profiles arise in equilibrium we define a set $A$ of possible states of $\omega$ by

$$
A=\left\{\omega \quad \mid \omega>1-\frac{\sigma}{\sqrt{2 \eta}}\right\} .
$$

On the set $A$ the relevant portfolio profile under ambiguity is the portfolio policy (26). Hence, on the set $A$ all agents in the model will participate in the equity market and asset prices will reflect the interaction of all market participants. On the complement $A^{c}$ the relevant portfolio profile under ambiguity is (28). This implies a very moderate exposure to equity. In fact, to first order 
such exposure is exactly zero. Hence, on this set asset prices will reflect primarily the portfolio behavior of the only participant in the equity market: the log utility investor. As a consequence, one agent in the economy will leave the equity market as soon as the endogenous state variable $\omega_{t}$ enters the set $A^{c}$. This implies a dynamic equity market participation in the model, which arises endogenously in dependence of the relevant state $\omega_{t}$ of the economy. We emphasize the endogeneity of the condition in the set $A$. In particular, such condition can be also not binding, for instance when volatility is very large relative to the amount of ambiguity $(\sigma / \sqrt{2 \eta} \geq 1)$. The characterization provided by the set $A$ extends to a dynamic setting the findings in Dow and Werlang (1992) that ambiguity aversion can imply naturally a limited equilibrium equity market participation even in the absence of transaction costs or other market frictions. Such distinct empirical prediction is supported by our setting (P2) of ambiguity aversion. Intuitively, the set $A$ is larger either when risk (measured by $\sigma$ ) is high, or when ambiguity (measured by $\eta$ ) is low. Indeed, while risk and standard risk aversion incentivate investors to trade in order to diversify risk, ambiguity reduces the incentives to trade because the output of the investment strategy becomes ambiguous.

The characterization of the general equilibrium under ambiguity aversion is provided by the next proposition.

Proposition 5 In the given production economy we have for the asymptotic equilibrium under the setting (P2) of ambiguity aversion:

1. Equilibrium interest rate on $A$

$$
r_{t}=\alpha-\sigma^{2}\left(1-\left(\gamma-\frac{\sqrt{2 \eta}}{\sigma}\right) \omega_{t}\right)+O^{2}(\gamma, \sqrt{\eta}),
$$

and on $A^{c}$

$$
r_{t}=\alpha-\frac{\sigma^{2}}{1-\omega_{t}}+O^{2}(\gamma, \sqrt{\eta})
$$

2. Optimal consumption asymptotics.

$$
C_{t}^{(1)}=\delta\left(1-\omega_{t}\right) S_{t} \quad, \quad C_{t}=\left(\delta-\gamma\left(\alpha-\sigma^{2} / 2-\delta\right)\right) \omega_{t} S_{t}+O^{2}(\gamma, \sqrt{\eta}) \quad .
$$


3. Optimal portfolios on $A$

$$
\begin{aligned}
w_{t}^{(1)} & =1-\left(\gamma-\frac{\sqrt{2 \eta}}{\sigma}\right) \omega_{t}+O^{2}(\gamma, \sqrt{\eta}), \\
w_{t} & =1+\left(\gamma-\frac{\sqrt{2 \eta}}{\sigma}\right)\left(1-\omega_{t}\right)+O^{2}(\gamma, \sqrt{\eta}),
\end{aligned}
$$

and on $A^{c}$

$$
w_{t}^{(1)}=\frac{1}{1-\omega_{t}}+O^{2}(\gamma, \sqrt{\eta}) \quad, \quad w_{t}=O^{2}(\gamma, \sqrt{\eta}) \quad .
$$

4. Cross-sectional wealth dynamics on $A$

$$
d \omega_{t}=\gamma \omega_{t}\left(1-\omega_{t}\right)\left(\alpha-\sigma^{2} / 2-\delta\right) d t+\left(\gamma-\frac{\sqrt{2 \eta}}{\sigma}\right) \omega_{t}\left(1-\omega_{t}\right) \sigma d Z_{t}+O^{2}(\gamma, \sqrt{\eta})
$$

and on $A^{c}$

$$
d \omega_{t}=\gamma \omega_{t}\left(1-\omega_{t}\right)\left[\alpha-\sigma^{2} / 2-\delta-\frac{1}{\gamma} \frac{\sigma^{2} \omega_{t}}{\left(1-\omega_{t}\right)^{2}}\right] d t-\omega_{t} \sigma^{2} d Z_{t}+O^{2}(\gamma, \sqrt{\eta})
$$

5. Capital stock dynamics.

$$
d S_{t}=\left[\alpha-\delta+\gamma\left(\alpha-\sigma^{2} / 2-\delta\right) \omega_{t}\right] S_{t} d t+\sigma S_{t} d Z_{t}+O^{2}(\gamma, \sqrt{\eta})
$$

The effect of ambiguity aversion on equilibrium consumption and capital stock dynamics is of higher order since the parameter $\eta$ does not affect the consumption policies (38). An indirect effect of ambiguity aversion arises, however, because of the different cross sectional wealth dynamics under ambiguity aversion. Equilibrium interest rates, optimal portfolios and cross-sectional wealth dynamics are influenced on the set $A$ directly by ambiguity aversion, since the parameter $\eta$ enters explicitly in (36), (39) and (42).

Compared to an economy without ambiguity $\left(\eta \rightarrow 0\right.$ and $\left.A^{c} \rightarrow \emptyset\right)$, we see from (36) that lower equilibrium interest rates are obtained on set $A$. On this set all agents hold a long position in equity and the contribution of ambiguity aversion to the lower interest rate is proportional to the volatility $\sigma$ of the capital stock dynamics. On the set $A$ the impact of ambiguity aversion on optimal equity holdings is inversely related to the volatility parameter $\sigma$. This highlights in a very simplified way the prudent equilibrium portfolio concern under ambiguity aversion in the setting (P2). However, remark that in general equilibrium the higher effective risk aversion $1-(\gamma-\sqrt{2 \eta} / \sigma)$ because of ambiguity aversion is state independent. An example of an equilibrium 
exchange economy where effective risk aversions under ambiguity aversion are state dependent to first order is presented in the next section.

On the set $A^{c}$ the log utility investor always borrows from the second agent her entire wealth, which is invested in a leveraged position in the risky asset. Such pattern arises independently of the coefficient of risk aversion $1-\gamma$ and the degree of ambiguity $\eta$ of the second agent. Therefore, on the set $A^{c}$ the $\log$ utility investor will always be leveraged in the risky asset. This implies a no risky asset investment zone, even in the absence of transaction costs or portfolio constraints, and produces a dynamic endogenous equity market participation in equilibrium. The set $A$ gives precise conditions under which such a situation is compatible with general equilibrium in the model: ambiguity parameterized by $\eta$ must be sufficiently large with respect to first order risk, as measured by volatility $\sigma$.

For $\omega_{t} \in A$ the basic structure of the effect of ambiguity aversion on the cross-sectional wealth dynamics (43) implies for $\gamma \neq 0$ a long run distribution of $\omega_{t}$ that is degenerated at $\omega_{t}=0$ or $\omega_{t}=1$ on $A$. The degeneracy of such distributions arises because we are looking only at a first order approximation of the relevant dynamics. In Dumas (1989), for a setting with no ambiguity aversion, stationary cross sectional wealth distributions are obtained. On set $A^{c}$, the drift and the volatility of the cross-sectional wealth dynamics (43) are altered in equilibrium. Moreover, for $\gamma<0$ the drift of $\omega_{t}$ can be always negative, if for instance $\alpha-\sigma^{2} / 2-\delta>0$, and the long run cross sectional wealth distribution will degenerate to 0 . However, if $\gamma>0$ - that is, the ambiguity averse investor is less risk averse than the log utility agent - the sign of the drift in the $\omega_{t}$ 's dynamics can change as $\omega_{t}$ approaches the boundary of the set $A^{c}$. In such case the long run distribution of $\omega_{t}$ can be stationary to first order for parameter choices such that the support of $\omega_{t}$ is in $A^{c}$, and limited equity market participation effects will persist as $t \rightarrow \infty$. 


\subsection{Exchange Economies}

We consider a generally incomplete heterogenous exchange economy where the risky asset is a claim on the aggregate endowment process $e_{t}$ with dynamics given by:

$$
\begin{aligned}
d e_{t} & =Y_{t} e_{t} d t+\sigma_{e} \sqrt{Y_{t}} e_{t} d Z_{t}^{e}, \\
d Y_{t} & =\lambda\left(\bar{Y}-Y_{t}\right) d t+\sqrt{Y_{t}} \sigma_{Y} d Z_{t}^{Y},
\end{aligned}
$$

where $\sigma_{e}, \lambda, \bar{Y}, \sigma_{Y}>0$, and $\left(Z_{t}^{e}\right),\left(Z_{t}^{Y}\right)$ are both standard Brownian motions in $\mathbb{R}$ with covariation $E\left(d Z_{t}^{e} d Z_{t}^{Y}\right)=\delta_{e Y} d t .\left(Y_{t}\right)$ is a mean reverting Bessel process that affects expected endowment returns and endowment returns volatilities. The cumulative return process of the risky asset is given by

$$
d P_{t}=\alpha_{t} P_{t} d t+P_{t}\left(\sigma_{P e t} d Z_{t}^{e}+\sigma_{P Y t} d Z_{t}^{Y}\right)
$$

where $\alpha_{t}, \sigma_{P e t}, \sigma_{P Y t}$ are drift and diffusion parameters to be determined in equilibrium. The locally riskless asset is a short term bond with price dynamics

$$
d B_{t}=r_{t} B_{t} d t
$$

where $r_{t}$ is an interest rate that has to be determined in equilibrium. Since for $\left|\delta_{e Y}\right| \neq 1$ the Brownian motions in the endowment and state dynamics are not perfectly correlated, we are in the context of a generically incomplete market. Hence, the relevant optimal investment policies for the setting (P2) of ambiguity aversion are those given in (23). In this case investors will never be able to avoid ambiguity by means of a suitable portfolio policy. This is a sharp difference with the complete market setting discussed in the previous section.

Definition 6 We call a process $\left(P_{t}, r_{t}, w_{t}, w_{t}^{(1)}, c_{t}, c_{t}^{(1)}\right)$ an asymptotic equilibrium under ambiguity aversion if

- The individual portfolio and consumption rules $w_{t}, w_{t}^{(1)}, c_{t}, c_{t}^{(1)}$ are asymptotically optimal, i.e. they satisfy (16) and (17) (they satisfy (22), (23)),

- The financial market and the good market clear: $w_{t} W_{t}+w_{t}^{(1)} W_{t}^{(1)}=W_{t}+W_{t}^{(1)}$ and $e_{t}=$ $C_{t}+C_{t}^{(1)}$. 
The relevant state variables for investors' decisions are expected endowment returns $Y_{t}$ and the cross-sectional wealth distribution $\omega_{t}$. Therefore, the function $g(Y, \omega)$ now depends on two arguments. Nevertheless, the same basic approximation methodology as in the above production economy example applies. Specifically, we can work with a function $g_{00}$ that depends only on the state variable $X_{t}:=Y_{t}$, yielding

$$
g_{0}(Y, \omega, \gamma, \vartheta)+O(\gamma, \vartheta)=g_{0,0}(Y)=g_{0}(Y, \omega, \gamma, \sqrt{\eta})+O(\gamma, \sqrt{\eta})
$$

where $g_{0,0}$ is uniquely determined by the representative-agent equilibrium of an economy populated by $\log$ utility agents. Such function $g_{0,0}$ is given in closed form in the next result.

Proposition 7 Under the given conditions:

$$
g_{0,0}(Y)=a+b Y+\ln (\delta) \quad,
$$

where

$$
a=\frac{\lambda\left(1-\frac{1}{2} \sigma_{e}^{2}\right) \bar{Y}}{\delta(\delta+\lambda)} \quad, \quad b=\frac{1-\frac{1}{2} \sigma_{e}^{2}}{\delta+\lambda}
$$

In particular, since $b>0$ for realistic parameter choices in the model we have $\partial g_{0,0} / \partial X>0$. The asymptotics for the general equilibrium under the settings (P1) and (P2) of ambiguity aversion are provided by the next proposition.

Proposition 8 Let

$$
G(Y)=\left(\sigma_{e}^{2}+2 \delta_{e Y} \sigma_{e} \sigma_{Y} b+\sigma_{Y}^{2} b^{2}\right) Y
$$

where $a$ and $b$ are given in (47) and define

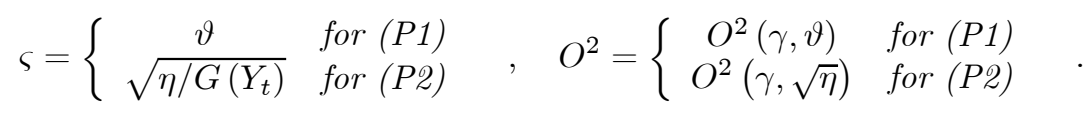

In the given exchange economy the following asymptotic expressions hold true:

1. Equilibrium stock prices process

$$
\begin{aligned}
P_{t}= & p\left(Y_{t}, \omega_{t}\right) e_{t} \\
p(Y, \omega)= & \frac{1}{\delta}(1+\gamma \omega(a+b Y))+O^{2} \\
\frac{d P_{t}+e_{t} d t}{P_{t}}= & {\left[\delta+Y_{t}\left(1-\gamma b \omega_{t}\left(\lambda+\delta-\delta_{e Y} \sigma_{e} \sigma_{Y}\right)\right)\right] d t } \\
& +\sigma_{e} \sqrt{Y_{t}} d Z_{t}^{e}+\gamma b \omega_{t} \sigma_{Y} \sqrt{Y_{t}} d Z_{t}^{Y}+O^{2}
\end{aligned}
$$

2. Equilibrium interest rate

$$
\begin{aligned}
r_{t}= & \left(\delta+\left(1-\gamma b \omega_{t}\left(\lambda+\delta-\delta_{e Y} \sigma_{e} \sigma_{Y}\right)\right) Y_{t}\right)-\left(\sigma_{e}^{2}+2 \sigma_{e} \gamma b \omega_{t} \sigma_{Y} \delta_{e Y}\right) Y_{t} \\
& +(\gamma-\varsigma)\left(\sigma_{e}^{2}+b \sigma_{e} \sigma_{Y} \delta_{e Y}\right) Y_{t} \omega_{t}+O^{2} .
\end{aligned}
$$


3. Optimal consumption and optimal portfolios

$$
\begin{aligned}
C_{t}^{(1)} & =\delta\left(1-\omega_{t}\right) S_{t} \\
C_{t} & =\delta\left(1-\gamma\left(a+b Y_{t}\right)\right) \omega_{t} S_{t}+O^{2} \\
w_{t}^{(1)} & =1-(\gamma-\varsigma)\left(1+b \frac{\sigma_{Y} \delta_{e Y}}{\sigma_{e}}\right) \omega_{t}+O^{2} \\
w_{t} & =1+(\gamma-\varsigma)\left(1+b \frac{\sigma_{Y} \delta_{e Y}}{\sigma_{e}}\right)\left(1-\omega_{t}\right)+O^{2} .
\end{aligned}
$$

4. Cross-sectional wealth dynamics.

$$
d \omega_{t}=\gamma \omega_{t}\left(1-\omega_{t}\right) b\left(\frac{\lambda \bar{Y}}{\delta}+Y_{t}\right) d t+(\gamma-\varsigma)\left(1-\omega_{t}\right) \sigma_{e} \sqrt{Y_{t}}\left(1+b \frac{\delta_{e Y} \sigma_{Y}}{\sigma_{e}}\right) d Z_{t}+O^{2}
$$

The marginal qualitative impact of ambiguity aversion on equilibrium portfolio holdings depends on whether

$$
\text { (i) } \gamma\left(1+b \frac{\sigma_{Y} \delta_{e Y}}{\sigma_{e}}\right)>0 \quad, \quad \text { (ii) } \gamma\left(1+b \frac{\sigma_{Y} \delta_{e Y}}{\sigma_{e}}\right)<0
$$

For instance, in case (i) ambiguity aversion reduces equilibrium leverage positions in the risky asset. This implies lower equilibrium interest rates (49) if and only if

$$
1+b \frac{\sigma_{Y} \delta_{e Y}}{\sigma_{e}}>0
$$

Thus, in the present economy interest rates can be in principle higher or lower under ambiguity aversion. Condition (53) is the requirement that intertemporal hedging motives do not dominate myopic demands in equilibrium portfolio holdings. ${ }^{15}$

The optimal portfolio behaviour (50) under the setting (P1) (i.e. $\varsigma=\vartheta$ ) is observationally equivalent to the one implied by an increased constant relative risk aversion $1-(\gamma-\vartheta)$. Hence, to first order optimal portfolios under the setting (P1) are state dependent only via cross sectional wealth $\omega_{t}$ : they are independent on the level of endowment expected growth rates and volatilities. The optimal portfolio behaviour (50) under the setting (P2) of ambiguity aversion (i.e. $\left.\varsigma=\sqrt{\eta / G\left(Y_{t}\right)}\right)$ is similar to the one implied by a state dependent increased risk aversion

\footnotetext{
15 For typical model parameter values condition (53) can be expected to be satisfied and interest rates will be lower under ambiguity aversion.
} 
$1-\left(\gamma-\sqrt{\eta / G\left(Y_{t}\right)}\right)$. To first order, this makes optimal portfolios dependent also on the level of endowment expected growth rates and volatilities. In particular, the largest state dependent risk aversions $1-(\gamma-\varsigma)$ arise for moderate values of the state variable $Y_{t}$, i.e. when endowment expected growth rates and volatilities are low. This highlights the very peculiar state dependent portfolio concern under ambiguity aversion in the setting (P2). An illustration of the equilibrium portfolio policy (51) is presented in Figure 3.

\section{Insert Figure 3 about here}

The panel on the left presents policies for $\eta=0.001$. The one on the right presents policies for $\eta=0.02$. For any given level of $Y$ optimal portfolios are linear in $\omega$. The slope of such a linear optimal policy increases dramatically as $Y \rightarrow 0$, and leads to significant short positions in equity for moderate levels of cross sectional wealth $\omega$ when $Y \rightarrow 0$.

The equilibrium effect of such portfolio behaviors on interest rates is described by (49). In particular, for $\varsigma=\vartheta$ the impact of ambiguity aversion in the setting (P1) is proportional to the variance $Y_{t} \sigma_{Y}^{2}$ of endowment returns. For $\varsigma=\sqrt{\eta / G\left(Y_{t}\right)}$ the effect on interest rates is proportional to $Y_{t} / \sqrt{G\left(Y_{t}\right)}$, i.e. the volatility of endowment returns. Since to first order the equity dynamics (48) is unaffected by ambiguity aversion, the impact on the arising equity premium is completely determined by the effect on equilibrium interest rates. Therefore, the setting (P1) of ambiguity aversion yields a contribution to equity premia that is linear in endowment variances, while setting (P2) implies a contribution that is linear in endowment volatilities. These are observationally distinct empirical predictions of our two settings of ambiguity aversion.

\section{Conclusions}

We explored analytically the empirical predictions of ambiguity aversion in heterogeneous agents economies with intermediate consumption and stochastic investment opportunity set using first order perturbation methods. To this end, we studied two intertemporal homothetic settings 
of ambiguity aversion motivated by two distinct robust control problems. One setting can be interpreted as a continuous time version of Recursive Multiple Priors Utility (RMPU). Our findings show that ambiguity aversion affects equilibria by acting more directly on equilibrium interest rates and portfolio holdings, rather than on equilibrium asset returns and aggregate consumption. The functional forms of optimal portfolios under the RMPU-unrelated robust control setting of ambiguity aversion are identical to those of a setting with no ambiguity but a higher constant relative risk aversion. Hence, such a setting of ambiguity aversion implies functional forms for equilibrium quantities like equity premia that are observationally equivalent to those of a setting with no ambiguity. In particular, the equity premium component due to ambiguity aversion is a second order function of volatility, i.e. it is proportional to return variances as in a setting with no ambiguity. The portfolio behavior under our RMPU setting of ambiguity aversion is instead similar to the one of a setting with no ambiguity but a higher and state dependent risk aversion, which is highly pronounced at moderate portfolio exposures. This feature causes quite prudent levels of equity market participations over a nontrivial set of states of the economy and an equity premium part due to ambiguity aversion that dominates when the exogenous random factors in the economy have low volatility. This last effect arises because the equity premium part due to ambiguity aversion in the RMPU setting is a first order function of volatility, i.e. it is proportional to volatility. Thus, the RMPU setting of ambiguity aversion can account for some distinct empirical predictions - like a limited equity market participation and ambiguity equity premia that dominate equity premia for small equity volatilities - which are unavailable under the RMPU-unrelated robust control setting. Such distinct empirical predictions of our more tractable setting of ambiguity aversion are consistent with other less tractable intertemporal settings of ambiguity aversion like the " $k$-ignorance" setting proposed in Chen and Epstein (2002). ${ }^{16}$

\footnotetext{
16 See for instance Chen and Epstein (2002), Section 5.
} 


\section{Appendix}

Proof of the non rectangularity of the set (12). Let:

$$
H:=\left\{h \quad: \quad E^{h}\left[\int_{0}^{\infty} e^{-\delta t} \frac{\left|h_{t}\right|^{2}}{2} d t\right] \leq k(\vartheta)\right\},
$$

and assume that it is a rectangular set, i.e.:

$$
H=\left\{h \quad: \quad h_{t} \in \Theta_{t}, t \geq 0\right\},
$$

where $\left(\Theta_{t}\right)_{t \geq 0}$ is a sequence of compact and convex subsets of $\mathbb{R}^{2}$ such that $\Theta_{t} \subset \mathcal{K}$, with $\mathcal{K}$ a compact subset of $\mathbb{R}^{2}$; cf. Chen and Epstein (2002), Section 2.4. Consider now a simple deterministic misspecification $h^{*}$ of the form

$$
h_{t}^{*}=\delta k(\vartheta) \exp \left(\frac{\delta}{2} t\right)
$$

Then, $h^{*}$ cannot belong to (55), since it is an unbounded function of $t$. However, $h^{*}$ belongs to (54). Indeed,

$$
E^{h}\left[\int_{0}^{\infty} e^{-\delta t} \frac{\left|h_{t}^{*}\right|^{2}}{2} d t\right]=\int_{0}^{\infty} e^{-\delta t} \frac{\left|h_{t}^{*}\right|^{2}}{2} d t=\frac{\delta}{2} k(\vartheta) \int_{0}^{\infty} e^{-\frac{\delta}{2} t} d t=k(\vartheta) .
$$

Hence, we have found a misspecification $h^{*}$ that is in (54) but not in (55). This shows that $H$ is not a rectangular set.

\section{Proof of Proposition 1. Define}

$$
\Lambda_{t}=\left[\begin{array}{cc}
\xi_{t} & 0 \\
\rho w_{t} W_{t} \sigma_{t} & \sqrt{1-\rho^{2}} w_{t} W_{t} \sigma_{t}
\end{array}\right],
$$

so that the joint dynamics of $Y_{t}=\left(X_{t}, W_{t}\right)^{\prime}$ can be written as

$$
d Y_{t}=\mu_{t} d t+\Lambda_{t} d Z_{t},
$$


where $\mu_{t}=\left(\zeta_{t}, w_{t} W_{t}\left(\alpha_{t}-r_{t}\right)+\left(r_{t} W_{t}-C_{t}\right)\right)^{\prime}$ and $Z$ is a standard Brownian motion in $\mathbb{R}^{2}$. The minimization with respect to $h$ in (6) gives (see for instance AHS (1998), p. 14)

$$
h^{H R}=-\frac{\vartheta}{\gamma J+\frac{1}{\delta}} \Lambda^{\prime}\left(\frac{\partial J}{\partial Y}\right)=-\frac{\vartheta}{\gamma J+\frac{1}{\delta}}\left(\begin{array}{c}
\xi J_{X}+\rho w W \sigma J_{W} \\
\sqrt{1-\rho^{2}} w W \sigma J_{W}
\end{array}\right),
$$

and the relevant single agent Hamilton Jacobi Bellman equation reads: ${ }^{17}$

$$
\begin{aligned}
0= & \sup _{c, w}\left\{\frac{(c W)^{\gamma}-1}{\gamma}-\delta J+(w W(\alpha-r)+(r W-c W)) J_{W}\right. \\
& +\frac{1}{2} w^{2} W^{2} \sigma^{2}\left(J_{W W}-\frac{\vartheta}{\gamma J+\frac{1}{\delta}} \cdot J_{W}^{2}\right) \\
& \left.+\zeta J_{X}+\frac{1}{2} \xi^{2}\left(J_{X X}-\frac{\vartheta}{\gamma J+\frac{1}{\delta}} J_{X}^{2}\right)+w W \sigma_{X P}\left(J_{X W}-\frac{\vartheta}{\gamma J+\frac{1}{\delta}} \cdot J_{W} J_{X}\right)\right\} .
\end{aligned}
$$

Homogeneity of $J$ implies the functional form

$$
J(W, X)=\frac{1}{\delta} \cdot \frac{\left(e^{g(X)} W\right)^{\gamma}-1}{\gamma}
$$

for some unknown function $g(X)$. Setting $\sigma_{X P}=\rho \xi \sigma$, for the optimal policies follows

$$
\begin{aligned}
c & =\frac{\left(J_{W}\right)^{\frac{1}{\gamma-1}}}{W}=\left(\frac{e^{\gamma g}}{\delta}\right)^{\frac{1}{\gamma-1}}, \\
w & =-\frac{1}{1-\frac{\vartheta}{\gamma J+\frac{1}{\delta}} \cdot \frac{J_{W}^{2}}{J_{W W}}} \cdot \frac{J_{W}}{W J_{W W}} \cdot\left(\frac{\alpha-r}{\sigma^{2}}+\frac{J_{W X}}{J_{W}} \frac{\sigma_{X P}}{\sigma^{2}}-\vartheta \frac{J_{X}}{\gamma J+\frac{1}{\delta}} \frac{\sigma_{X P}}{\sigma^{2}}\right) \\
& =\frac{1}{1-(\gamma-\vartheta)} \cdot\left(\frac{\alpha-r}{\sigma^{2}}+(\gamma-\vartheta) \frac{\partial g}{\partial X} \cdot \frac{\sigma_{X P}}{\sigma^{2}}\right) .
\end{aligned}
$$

Writing $g=g_{0}+\gamma g_{1}+\vartheta g_{2}+O^{2}(\gamma, \vartheta)$ and expanding (60), (61) up to first order in $(\gamma, \vartheta)$ proves the proposition.

Proof of Proposition 2 . The minimization with respect to $h$ in (10) gives (see for instance AHS (1998), p. 22)

$$
\begin{aligned}
h^{C R} & =-\frac{\sqrt{2 \eta}}{\left(\frac{\partial J^{\prime}}{\partial Y} \Lambda \Lambda^{\prime} \frac{\partial J}{\partial Y}\right)^{\frac{1}{2}}} \Lambda^{\prime} \frac{\partial J}{\partial Y} \\
& =-\frac{\sqrt{2 \eta}}{\left(W^{2} J_{W}^{2} \sigma^{2}+\xi^{2} J_{X}^{2}+2 \sigma_{X P} W J_{X} J_{W}\right)^{\frac{1}{2}}}\left(\begin{array}{c}
\xi J_{X}+\rho w W \sigma J_{W} \\
\sqrt{1-\rho^{2}} w W \sigma J_{W}
\end{array}\right) .
\end{aligned}
$$

17 We use subscripts to denote partial derivatives with respect to the relevant argument. 
Hence, the single agent HJB equation is

$$
\begin{aligned}
0= & \sup _{c, w}\left\{\frac{(c W)^{\gamma}-1}{\gamma}-\delta J+(w W(\alpha-r)+(r W-c W)) J_{W}+\frac{1}{2} w^{2} W^{2} \sigma^{2} J_{W W}\right. \\
& \left.+\zeta J_{X}+\frac{1}{2} \xi^{2} J_{X X}+w W \sigma_{X P} J_{X W}-\sqrt{2 \eta}\left[w^{2} W^{2} \sigma^{2} J_{W}^{2}+\xi^{2} J_{X}^{2}+2 w W \sigma_{X P} J_{W} J_{X}\right]^{\frac{1}{2}}\right\} .
\end{aligned}
$$

Homogeneity of $J$ implies an homogeneous functional form of the type (14). Hence, the implied optimal policies are given by

$$
\begin{aligned}
c & =\frac{\left(J_{W}\right)^{\frac{1}{\gamma-1}}}{W}=\left(\frac{e^{\gamma g}}{\delta}\right)^{\frac{1}{\gamma-1}} \\
w & =\frac{1}{1-\sqrt{\frac{2 \eta}{\Gamma(w)}} \frac{J_{W}^{2}}{J_{W W}}} \cdot \frac{1}{1-\gamma}\left(\frac{\alpha-r}{\sigma^{2}}+\gamma \frac{\partial g}{\partial X} \cdot \frac{\sigma_{X P}}{\sigma^{2}}-\sqrt{\frac{2 \eta}{\Gamma(w)}} J_{X} \frac{\sigma_{X P}}{\sigma^{2}}\right),
\end{aligned}
$$

where

$$
\Gamma(w)=W^{2} J_{W}^{2}\left(\sigma^{2} w^{2}+\frac{\xi^{2} J_{X}^{2}}{W^{2} J_{W}^{2}}+2 w \sigma_{X P} \frac{J_{X}}{W J_{W}}\right)=W^{2} J_{W}^{2} G(w)
$$

Using the last expression for $\Gamma$, the optimal policy (65) reads more compactly

$$
w=\frac{1}{1-\left(\gamma-\sqrt{\frac{2 \eta}{G(w)}}\right)} \cdot\left(\frac{\alpha-r}{\sigma^{2}}+\left(\gamma-\sqrt{\frac{2 \eta}{G(w)}}\right) \frac{\partial g}{\partial X} \frac{\sigma_{X P}}{\sigma^{2}}\right)
$$

Hence, the optimal investment policy is the solution of an implicit equation in $w$. To expand (64), (66), up to first order, we first write

$$
\begin{aligned}
& w(X)=\frac{\alpha-r}{\sigma^{2}}+\gamma w_{1}(X)+\sqrt{2 \eta} w_{2}(X)+O^{2}(\gamma, \sqrt{\eta}) \\
& g(X)=g_{0}(X)+\gamma g_{1}(X)+\sqrt{2 \eta} g_{2}(X)+O^{2}(\gamma, \sqrt{\eta})
\end{aligned}
$$

Expanding (64), (66) while making use of (67) and (68) concludes the proof of the Proposition.

Proof of Proposition 3. We discuss the case $\rho=1$ since the case $\rho=-1$ can be treated in the same way. In this case, the relevant HJB equation (63) for problem (10) becomes:

$$
\begin{aligned}
0= & \sup _{c, w}\left\{\frac{(c W)^{\gamma}-1}{\gamma}-\delta J+(w W(\alpha-r)+(r W-c W)) J_{W}+\frac{1}{2} w^{2} W^{2} \sigma^{2} J_{W W}\right. \\
& \left.+\zeta J_{X}+\frac{1}{2} \xi^{2} J_{X X}+w W \sigma_{X P} J_{X W}-\sqrt{2 \eta}\left|w W \sigma J_{W}+\xi J_{X}\right|\right\}
\end{aligned}
$$


Homogeneity of $J$ implies an homogeneous functional form of the type (14). Hence, the optimal consumption policy is given by:

$$
c=\left(\frac{e^{\gamma g}}{\delta}\right)^{\frac{1}{\gamma-1}} .
$$

A first order expansion of $g$ then gives the first order consumption policy. To express the optimal investment policies we distinguish two cases:

$$
\text { (i) } w W \sigma J_{W}+\xi J_{X} \geq 0 \quad ; \quad \text { (ii) } \quad w W \sigma J_{W}+\xi J_{X} \leq 0 \quad \text {. }
$$

For case (i), the HJB equation reads

$$
\begin{aligned}
0= & \sup _{c, w}\left\{\frac{(c W)^{\gamma}-1}{\gamma}-\delta J+(w W(\alpha-r)+(r W-c W)) J_{W}+\frac{1}{2} w^{2} W^{2} \sigma^{2} J_{W W}\right. \\
& \left.+\zeta J_{X}+\frac{1}{2} \xi^{2} J_{X X}+w W \sigma_{X P} J_{X W}-\sqrt{2 \eta}\left(w W \sigma J_{W}+\xi J_{X}\right)+\lambda\left(w W \sigma J_{W}+\xi J_{X}\right)\right\} \\
= & \sup _{c, w}\left\{\frac{(c W)^{\gamma}-1}{\gamma}-\delta J+(w W(\alpha-r-(\sqrt{2 \eta}-\lambda) \sigma)+(r W-c W)) J_{W}\right. \\
& \left.+\frac{1}{2} w^{2} W^{2} \sigma^{2} J_{W W}+(\zeta-\xi(\sqrt{2 \eta}-\lambda)) J_{X}+\frac{1}{2} \xi^{2} J_{X X}+w W \sigma_{X P} J_{X W}\right\}
\end{aligned}
$$

where $\lambda \geq 0$ is a Lagrange multiplier. The optimal investment policy is

$$
\begin{aligned}
w & =-\frac{1}{\sigma^{2} W J_{W W}}\left[(\alpha-r-(\sqrt{2 \eta}-\lambda) \sigma) J_{W}+\sigma_{X P} J_{X W}\right] \\
& =\frac{1}{\sigma^{2}(1-\gamma)}\left(\alpha-r-(\sqrt{2 \eta}-\lambda) \sigma+\gamma \xi \sigma \frac{\partial g}{\partial X}\right),
\end{aligned}
$$

using the functional form (14). Slackness implies:

$$
0=\lambda\left(w W \sigma J_{W}+\xi J_{X}\right)=\frac{\lambda}{\delta}\left(w \sigma+\xi \frac{\partial g}{\partial X}\right) W^{\gamma} .
$$

From (72) the Lagrange multiplier $\lambda$ is given by

$$
\begin{aligned}
\lambda \sigma & =\sigma^{2}(1-\gamma) w-(\alpha-r)+\sqrt{2 \eta} \sigma-\gamma \xi \sigma \frac{\partial g}{\partial X} \\
& =-(1-\gamma) \xi \sigma \frac{\partial g}{\partial X}-(\alpha-r)+\sqrt{2 \eta} \sigma-\gamma \xi \sigma \frac{\partial g}{\partial X} \\
& =-\left(\alpha-r-\sqrt{2 \eta} \sigma+\xi \sigma \frac{\partial g}{\partial X}\right) \geq 0,
\end{aligned}
$$

using (73). Hence, the constraint is binding if

$$
\alpha-r+\xi \sigma \frac{\partial g}{\partial X} \leq \sqrt{2 \eta} \sigma
$$


i.e. if

$$
\frac{1}{\sigma^{2}(1-\gamma)}\left(\alpha-r-\sqrt{2 \eta} \sigma+\gamma \xi \sigma \frac{\partial g}{\partial X}\right) \leq-\frac{\xi}{\sigma} \frac{\partial g}{\partial X}
$$

In the opposite case $\lambda=0$ and the optimal policy is given by (72). In the case of a binding constraint, slackness implies an optimal policy given by

$$
w=-\frac{\xi}{\sigma} \frac{\partial g}{\partial X}
$$

The optimal policy for the case (ii) follows analogously. To get a first order expression for the optimal investment policy it is sufficient to note that

$$
\sqrt{2 \eta}\left|w W \sigma J_{W}+\xi J_{X}\right|=\frac{\sqrt{2 \eta}}{\delta}\left|w \sigma+\xi \frac{\partial g}{\partial X}\right| W^{\gamma}=\frac{\sqrt{2 \eta}}{\delta}\left|w \sigma+\xi \frac{\partial g_{0}}{\partial X}\right| W^{\gamma}+O^{2}(\gamma, \sqrt{2 \eta})
$$

By inserting the approximation (74) in the HJB equation (69) the implied optimality conditions can be then obtained up to first order in $\gamma, \sqrt{2 \eta}$, with the same approach used above to compute the optimal portfolio policy. Solving such optimality condition for the investment policy gives the result.

Proof of the result in Footnote 10. Using formula (19) in Kogan and Uppal (2000) it follows

$$
\begin{aligned}
g_{0}(X) & =\ln (\delta)-1+E\left[\int_{0}^{\infty} e^{-\delta t}\left(r+\frac{1}{2}\left(\frac{\alpha_{t}-r}{\sigma}\right)^{2}\right) d t \mid X_{0}=X\right] \\
& =\ln (\delta)-1+\left[\int_{0}^{\infty} e^{-\delta t}\left(r+\frac{1}{2} E\left(X_{t}^{2} \mid X_{0}=X\right)\right) d t\right]
\end{aligned}
$$

Since

$$
\begin{aligned}
E\left(X_{t}^{2} \mid X_{0}=X\right) & =\operatorname{Var}\left(X_{t} \mid X_{0}=X\right)+\left(E\left(X_{t} \mid X_{0}=X\right)\right)^{2} \\
& =\xi^{2} \cdot \frac{1-e^{-2 \lambda t}}{2 \lambda}+\left[e^{-\lambda t}\left(X+\bar{X}\left(e^{\lambda t}-1\right)\right)\right]^{2}
\end{aligned}
$$


a final integration gives

$$
\begin{aligned}
g_{0}(X)= & \ln (\delta)-1+\int_{0}^{\infty} e^{-\delta t}\left(r+\frac{\xi^{2}}{2} \cdot \frac{1-e^{-2 \lambda t}}{2 \lambda}+\frac{1}{2}\left[e^{-\lambda t}\left(X+\bar{X}\left(e^{\lambda t}-1\right)\right)\right]^{2}\right) d t \\
= & \ln (\delta)-1+\int_{0}^{\infty} e^{-\delta t}\left(r+\frac{\xi^{2}}{2} \cdot \frac{1-e^{-2 \lambda t}}{2 \lambda}+\frac{\left(e^{-\lambda t} \bar{X}\left(e^{\lambda t}-1\right)\right)^{2}}{2}\right) d t \\
& +X \cdot \bar{X} \int_{0}^{\infty}\left(e^{-\delta t}\left(e^{\lambda t}-1\right)\right) d t+\frac{X^{2}}{2} \cdot \int_{0}^{\infty} e^{-\delta t} e^{-2 \lambda t} d t .
\end{aligned}
$$

By computing these expectations explicitly, the claim of the proposition is obtained.

Notation: In the sequel we adopt the symbol $\doteq$ to denote equality up to terms of order $O^{2}(\gamma, \vartheta)$ and $O^{2}(\gamma, \sqrt{\eta})$.

Proof of Proposition 5. We have:

$$
g_{0}(X, \gamma, \eta)+O(\|(\gamma, \sqrt{\eta})\|)=g_{00}=\log (\delta)-1+\frac{\alpha-\sigma^{2} / 2}{\delta} .
$$

Aggregate market clearing implies

$$
1 \doteq w_{t}^{(1)}+\left(w_{t}-w_{t}^{(1)}\right) \omega_{t}
$$

Since in the present setting $\frac{\partial g_{00}(X)}{\partial X}=0$, the complete markets asymptotics for optimal investment in Corollary 3 implies

$$
w_{t} \doteq\left\{\begin{array}{cc}
\frac{1}{\sigma^{2}(1-\gamma)}\left(\alpha-r_{t}-\sqrt{2 \eta} \sigma\right) & , \text { if } \alpha-r_{t}-\sqrt{2 \eta} \sigma \geq 0 \\
\frac{1}{\sigma^{2}(1-\gamma)}\left(\alpha-r_{t}+\sqrt{2 \eta} \sigma\right) & , \text { if } \alpha-r_{t}+\sqrt{2 \eta} \sigma \leq 0 \\
0 & \text { otherwise }
\end{array} .\right.
$$

In general equilibrium, the relevant cases are:

$$
\text { Case 1: } \quad \frac{\alpha-r_{t}}{\sigma}>\sqrt{2 \eta} \quad, \quad \text { Case } 2: \quad 0<\frac{\alpha-r_{t}}{\sigma} \leq \sqrt{2 \eta}
$$

For Case 1 follows

$$
1 \doteq w_{t}^{(1)}+\left(w_{t}-w_{t}^{(1)}\right) \omega_{t} \doteq \frac{\left(\alpha-r_{t}\right)\left(1-\gamma\left(1-\omega_{t}\right)\right)-\sqrt{2 \eta} \sigma \omega_{t}}{(1-\gamma) \sigma^{2}}
$$


implying

$$
\frac{\alpha-r_{t}}{\sigma} \doteq \frac{(1-\gamma) \sigma+\sqrt{2 \eta} \omega_{t}}{1-\gamma\left(1-\omega_{t}\right)}
$$

Hence, for Case 1

$$
\frac{\alpha-r_{t}}{\sigma}>\sqrt{2 \eta} \Longleftrightarrow(1-\gamma) \sigma+\sqrt{2 \eta} \omega_{t}>(1-\gamma) \sqrt{2 \eta}+\gamma \sqrt{2 \eta} \omega_{t} \Longleftrightarrow \omega_{t}>1-\frac{\sigma}{\sqrt{2 \eta}}
$$

Solving for $r_{t}$, we get

$$
r_{t} \doteq \alpha-\sigma \frac{(1-\gamma) \sigma+\sqrt{2 \eta} \omega_{t}}{1-\gamma\left(1-\omega_{t}\right)} \doteq \alpha-\sigma^{2}\left(1-\left(\gamma-\frac{\sqrt{2 \eta}}{\sigma}\right) \omega_{t}\right)
$$

Similarly, for Case 2

$$
1 \doteq w_{t}^{(1)}\left(1-\omega_{t}\right) \doteq \frac{\alpha-r_{t}}{\sigma^{2}}\left(1-\omega_{t}\right)
$$

which implies

$$
r_{t}=\alpha-\frac{\sigma^{2}}{1-\omega_{t}}
$$

This proves 1 . of the proposition.

The consumption asymptotics in Proposition 2 (which are valid also for the complete markets case) implies

$$
c_{t}^{(1)}=\delta \quad, \quad c_{t} \doteq \delta-\gamma \delta\left(g_{0}(X)-\log (\delta)\right) \doteq \delta-\gamma\left(\alpha-\sigma^{2} / 2-\delta\right)
$$

Moreover, from (75) and (76)

$$
w_{t}^{(1)}=\frac{\alpha-r_{t}}{\sigma^{2}} \doteq 1-\left(\gamma-\frac{\sqrt{2 \eta}}{\sigma}\right) \omega_{t}
$$

in Case 1, and

$$
w_{t}^{(1)}=\frac{1}{1-\omega_{t}},
$$

in Case 2. In the same vain (again by $(75),(76)$ )

$$
w_{t} \doteq \frac{1}{\sigma^{2}(1-\gamma)}\left(\alpha-r_{t}-\sqrt{2 \eta} \sigma\right) \doteq 1+\left(\gamma-\frac{\sqrt{2 \eta}}{\sigma}\right)\left(1-\omega_{t}\right)
$$


in Case 1 , and $w_{t} \doteq 0$ otherwise. This proves 2 . of the proposition. To prove 3 , , we consider as in the previous robustness setting, the Itô's dynamics of $\omega_{t}$

$$
\begin{aligned}
d \omega_{t} \doteq & \omega_{t}\left(1-\omega_{t}\right)\left[\left(w_{t}-w_{t}^{(1)}\right)\left[\left(\alpha-r_{t}\right)-\left(\omega_{t} w_{t}+\left(1-\omega_{t}\right) w_{t}^{(1)}\right) \sigma_{t}^{2}\right]\right. \\
& \left.-\left(c_{t}-c_{t}^{(1)}\right)\right] d t+\omega_{t}\left(1-\omega_{t}\right)\left(w_{t}-w_{t}^{(1)}\right) \sigma d Z_{t} .
\end{aligned}
$$

and distinguish again the two Cases considered above. We have

$$
-\left(c_{t}-c_{t}^{(1)}\right) \doteq \gamma\left(\alpha-\sigma^{2} / 2-\delta\right)
$$

and

$$
\begin{aligned}
\left(w_{t}-w_{t}^{(1)}\right) \sigma & \doteq \sigma \gamma-\sqrt{2 \eta} \quad, \quad \text { Case } 1 \\
\left(w_{t}-w_{t}^{(1)}\right) \sigma & \doteq-\frac{\sigma}{1-\omega_{t}} \quad, \quad \text { Case } 2 .
\end{aligned}
$$

Using the financial markets clearing condition we have

$$
\left(\alpha-r_{t}\right)-\left(\omega_{t} w_{t}+\left(1-\omega_{t}\right) w_{t}^{(1)}\right) \sigma^{2} \doteq\left(\alpha-r_{t}\right)-\sigma^{2}
$$

implying

$$
\left(w_{t}-w_{t}^{(1)}\right)\left[\left(\alpha-r_{t}\right)-\left(\omega_{t} w_{t}+\left(1-\omega_{t}\right) w_{t}^{(1)}\right) \sigma^{2}\right] \doteq 0
$$

for Case 1. On the other hand, for Case 2 it follows

$$
\left(w_{t}-w_{t}^{(1)}\right)\left[\left(\alpha-r_{t}\right)-\sigma^{2}\right] \doteq-\frac{1}{1-\omega_{t}}\left(\frac{\sigma^{2}}{1-\omega_{t}}-\sigma^{2}\right)=-\frac{\sigma^{2} \omega_{t}}{\left(1-\omega_{t}\right)^{2}}
$$

Putting the results together we proved 3. We remark that this last equation also implies an asymptotic contribution of order no less than 2 for the optimal portfolio policies to the drift of the cross sectional wealth dynamics in the Cases 1 . and 2.

To prove 4 , we get from (77)

$$
\begin{aligned}
d S_{t} & \doteq\left(\alpha S_{t}-\left(C_{t}+C_{t}^{(1)}\right)\right) d t+\sigma S_{t} d Z_{t} \\
& \doteq\left[\alpha S_{t}-\left(\left(\delta-\gamma\left(\alpha-\sigma^{2} / 2-\delta\right)\right) W_{t}+\delta W_{t}^{(1)}\right)\right] d t+\sigma S_{t} d Z_{t} \\
& \doteq\left[\alpha-\delta+\gamma\left(\alpha-\sigma^{2} / 2-\delta\right) \omega_{t}\right] S_{t} d t+\sigma S_{t} d Z_{t}
\end{aligned}
$$


This proves 4 and the whole proposition.

Proof of Proposition 7. The value function of the representative log-utility agents is

$$
\frac{1}{\delta}\left(\log \left(W_{0}\right)+g_{0,0}\left(Y_{0}\right)\right)=E_{0}\left[\int_{0}^{\infty} e^{-\delta t} \log \left(e_{t}\right) d t\right]
$$

Computing the expectation on the RHS of (78) it follows

$$
\begin{aligned}
E_{0}\left[\int_{0}^{\infty} e^{-\delta t} \log \left(e_{t}\right) d t\right] & =E_{0}\left[\int_{0}^{\infty} e^{-\delta t}\left(\log \left(e_{0}\right)+\left(1-\frac{1}{2} \sigma_{e}^{2}\right) \int_{0}^{t} Y_{s} d s\right) d t\right] \\
& =\log \left(e_{0}\right) \int_{0}^{\infty} e^{-\delta t} d t+\left(1-\frac{1}{2} \sigma_{e}^{2}\right) \int_{0}^{\infty} e^{-\delta t}\left(\int_{0}^{t} E_{0}\left(Y_{s}\right) d s\right) d t
\end{aligned}
$$

Using the formula

$$
E_{0}\left(Y_{s}\right)=\bar{Y}+\left(Y_{0}-\bar{Y}\right) e^{-\lambda s}
$$

we then have

$$
\begin{aligned}
E_{0}\left[\int_{0}^{\infty} e^{-\delta t} \log \left(e_{t}\right) d t\right] & =\frac{1}{\delta} \ln \left(e_{0}\right)+\left(1-\frac{1}{2} \sigma_{e}^{2}\right) \int_{0}^{\infty} e^{-\delta t}\left(\bar{Y} t+\left(Y_{0}-\bar{Y}\right) \int_{0}^{t} e^{-\lambda s} d s\right) d t \\
& =\frac{1}{\delta} \ln \left(e_{0}\right)+\frac{1-\frac{1}{2} \sigma_{e}^{2}}{\delta(\delta+\lambda)}\left(Y_{0}+\frac{\lambda \bar{Y}}{\delta}\right)
\end{aligned}
$$

At the same time, $W_{0}$ is the aggregate wealth of the economy, which is equal to the price of the stock, $\delta^{-1} e_{0}$. Thus, $g_{0,0}\left(Y_{0}\right)=a+b Y_{0}+\ln (\delta)$, where

$$
a=\frac{\lambda\left(1-\frac{1}{2} \sigma_{e}^{2}\right)}{\delta(\delta+\lambda)} \bar{Y} \quad, \quad b=\frac{1-\frac{1}{2} \sigma_{e}^{2}}{\delta+\lambda}
$$

Proof of Proposition 8. To prove 1, we note that in our economy $W_{t}+W_{t}^{(1)}=P_{t}$ and

$$
W_{t}=\omega_{t} P_{t} \quad, \quad W_{t}^{(1)}=\left(1-\omega_{t}\right) P_{t}
$$

Using the consumption asymptotics $(16)$, with $g_{0}$ replaced by $g_{00}$, aggregate good markets clearing implies

$$
\begin{aligned}
\frac{e_{t}}{\delta} & \doteq \frac{1}{\delta}\left(c_{t}+c_{t}^{(1)}\right) \doteq \frac{1}{\delta}\left(\delta\left(1-\omega_{t}\right)+\delta\left(1-\gamma\left(g_{0,0}\left(X_{t}\right)-\log (\delta)\right)\right) \omega_{t}\right) P_{t} \\
& \doteq\left(1-\gamma\left(g_{0,0}\left(X_{t}\right)-\log (\delta)\right) \omega_{t}\right) P_{t},
\end{aligned}
$$


that is:

$$
P_{t} \doteq \frac{e_{t}}{\delta}\left(1+\gamma\left(a+b Y_{t}\right) \omega_{t}\right)
$$

Defining $p_{t}=p\left(Y_{t}, \omega_{t}\right)=\frac{1}{\delta}\left(1+\gamma\left(a+b Y_{t}\right) \omega_{t}\right)$, it follows

$$
\begin{aligned}
\frac{d P_{t}}{P_{t}} & \doteq Y_{t} d t+\gamma b \omega_{t}\left(d Y_{t}+\left[d Y_{t}, \frac{d e_{t}}{e_{t}}\right]\right)+\sigma_{e} \sqrt{Y_{t}} d Z_{t}^{e} \\
& =\left(Y_{t}+\gamma b \omega_{t}\left(\lambda\left(\bar{Y}-Y_{t}\right)+Y_{t} \delta_{e Y} \sigma_{e} \sigma_{Y}\right)\right) d t+\sigma_{e} \sqrt{Y_{t}} d Z_{t}^{e}+\gamma b \omega_{t} \sigma_{Y} \sqrt{Y_{t}} d Z_{t}^{Y}
\end{aligned}
$$

The cumulative return then is

$$
\begin{aligned}
\frac{d P_{t}+e_{t} d t}{P_{t}} \doteq & \left(Y_{t}+\gamma b \omega_{t}\left(\lambda\left(\bar{Y}-Y_{t}\right)+Y_{t} \delta_{e Y} \sigma_{e} \sigma_{Y}\right)\right) d t+\delta\left(1-\gamma \omega_{t}\left(a+b Y_{t}\right)\right) d t \\
& +\sigma_{e} \sqrt{Y_{t}} d Z_{t}^{e}+\gamma b \omega_{t} \sigma_{Y} \sqrt{Y_{t}} d Z_{t}^{Y} \\
\doteq & \left(Y_{t}+\delta-\gamma b \omega_{t} Y_{t}\left(\lambda+\delta-\delta_{e Y} \sigma_{e} \sigma_{Y}\right)\right) d t+\sigma_{e} \sqrt{Y_{t}} d Z_{t}^{e}+\gamma b \omega_{t} \sigma_{Y} \sqrt{Y_{t}} d Z_{t}^{Y} \\
= & \left(\delta+Y_{t}\left(1-\gamma b \omega_{t}\left(\lambda+\delta-\delta_{e Y} \sigma_{e} \sigma_{Y}\right)\right)\right) d t+\sigma_{e} \sqrt{Y_{t}} d Z_{t}^{e}+\gamma b \omega_{t} \sigma_{Y} \sqrt{Y_{t}} d Z_{t}^{Y} .
\end{aligned}
$$

This proves 1 . To prove 2 we first notice that the market price of risk $\phi$ under ambiguity is given by:

$$
\phi(Y, \omega)=\phi_{00}(Y, \omega)+\gamma \phi_{01}(Y, \omega)+\sqrt{2 \eta} \phi_{02}(Y, \omega)+O^{2}(\gamma, \sqrt{\eta})
$$

where

$$
\phi_{00}(Y, \omega)=\sigma_{e} \sqrt{Y},
$$

is the market price of risk in a standard homogeneous economy populated by log-utility agents. Hence:

$$
\sqrt{\frac{\eta}{G_{0}}} \doteq \sqrt{\frac{\eta}{G(Y)}}
$$

where

$$
G(Y)=\phi_{00}^{2}(Y)+2 \phi_{00}(Y) \delta_{e Y} \sigma_{Y} \sqrt{Y} b+\left(\sigma_{Y} \sqrt{Y} b\right)^{2}=Y\left(\sigma_{e}^{2}+2 \delta_{e Y} \sigma_{e} \sigma_{Y} b+\sigma_{Y}^{2} b^{2}\right)
$$

Defining

$$
\varsigma=\left\{\begin{array}{cc}
\vartheta & \text { for robustness } \\
\sqrt{\eta / G\left(Y_{t}\right)} & \text { for ambiguity }
\end{array}\right.
$$


financial markets clearing gives

$$
r_{t} \doteq \alpha_{t}+e_{t}-\sigma_{t}^{2}+(\gamma-\varsigma)\left(\sigma_{t}^{2}+\frac{\partial g_{0,0}\left(Y_{t}\right)}{\partial X} \sigma_{X P}\right) \omega_{t} \doteq \alpha_{t}+e_{t}-\sigma_{t}^{2}+(\gamma-\varsigma)\left(\sigma_{t}^{2}+b \sigma_{X P}\right) \omega_{t}
$$

Using the above cumulative return dynamics this leads to

$$
\begin{aligned}
r_{t} \doteq & \left(\delta+\left(1-\gamma b \omega_{t}\left(\lambda+\delta-\delta_{e Y} \sigma_{e} \sigma_{Y}\right)\right) Y_{t}\right)-\left(\sigma_{e}^{2}+2 \sigma_{e} \gamma b \omega_{t} \sigma_{Y} \delta_{e Y}\right) Y_{t} \\
& +(\gamma-\varsigma)\left(\sigma_{e}^{2}+b \sigma_{e} \sigma_{Y} \delta_{e Y}\right) Y_{t} \omega_{t} .
\end{aligned}
$$

which proves 2 .

The first part of Claim 3 is based on the optimum consumption asymptotics under robustness or ambiguity together with (46):

$$
c_{t}^{(1)}=\delta \quad, \quad c_{t} \doteq \delta\left(1-\gamma\left(g_{00}\left(Y_{t}\right)-\log (\delta)\right)\right) \doteq \delta\left(1-\gamma\left(a+b Y_{t}\right)\right)
$$

Moreover,

$$
w^{(1)} \doteq \frac{\alpha_{t}+e_{t}-r_{t}}{\sigma_{t}^{2}} \doteq 1-(\gamma-\varsigma)\left(1+b \frac{\sigma_{Y} \delta_{e Y}}{\sigma_{e}}\right) \omega_{t}
$$

and

$$
\begin{aligned}
w & \doteq w^{(1)}(1+\gamma-\varsigma)+(\gamma-\varsigma) \frac{\partial g_{0,0}\left(Y_{t}\right)}{\partial X} \frac{\sigma_{X P}}{\sigma_{t}^{2}} \\
& \doteq 1+(1+\gamma-\varsigma)-(\gamma-\varsigma)\left(1+b \frac{\sigma_{Y} \delta_{e Y}}{\sigma_{e}}\right) \omega_{t}+(\gamma-\varsigma) b \frac{\sigma_{Y} \delta_{e Y}}{\sigma_{e}} \\
& \doteq 1+(\gamma-\varsigma)\left(1+b \frac{\sigma_{Y} \delta_{e Y}}{\sigma_{e}}\right)\left(1-\omega_{t}\right)
\end{aligned}
$$

This proves 3 .

We next consider 4 . The equilibrium cross-sectional wealth dynamics implied by Itô's Lemma for the given exchange economy are of the form

$$
\begin{aligned}
d \omega_{t}= & \omega_{t}\left(1-\omega_{t}\right)\left[\left(w_{t}-w_{t}^{(1)}\right)\left[\left(\alpha_{t}+e_{t}-r_{t}\right)-\sigma_{t}^{2}\right]-\left(c_{t}-c_{t}^{(1)}\right)\right] d t \\
& +\omega_{t}\left(1-\omega_{t}\right)\left(w_{t}-w_{t}^{(1)}\right) \sigma_{t} d Z_{t}
\end{aligned}
$$

Moreover

$$
\left(w-w_{t}^{(1)}\right) \sigma_{t} \doteq(\gamma-\varsigma)\left(1+b \frac{\sigma_{Y} \delta_{e Y}}{\sigma_{e}}\right) \sigma_{e} \sqrt{Y_{t}}
$$


and

$$
-\left(c_{t}-c_{t}^{(1)}\right) \doteq \delta \gamma\left(a+b Y_{t}\right)
$$

Finally, using the results in the proof of 2 we have

$$
\left(\alpha_{t}+e_{t}-r_{t}\right)-\sigma_{t}^{2} \doteq-(\gamma-\varsigma)\left(\sigma_{t}^{2}+\frac{\partial g_{0,0}\left(Y_{t}\right)}{\partial X} \sigma_{X P}\right) \omega_{t} \doteq-(\gamma-\varsigma)\left(\sigma_{e}^{2}+b \sigma_{e} \sigma_{Y} \delta_{e Y}\right) Y_{t} \omega_{t}
$$

yielding

$$
\left(w_{t}-w_{t}^{(1)}\right)\left[\left(\alpha_{t}+e_{t}-r_{t}\right)-\left(\omega_{t} w_{t}+\left(1-\omega_{t}\right) w_{t}^{(1)}\right) \sigma_{t}^{2}\right] \doteq 0
$$

This proves 4 . 


\section{References}

Anderson, E. W., L. P. Hansen, and T. J. Sargent, 1998. Risk and Robustness in General Equilibrium, Manuscript, University of Chicago.

Anderson, E. W., L. P. Hansen, and T. J. Sargent, 2003. Robustness, Detection and the Price of Risk, Manuscript, University of Chicago

Chen, Z. and L. G. Epstein, 2002. Ambiguity, Risk and Asset Returns in Continuous Time, Econometrica, 70, 1403-1443.

Dow, J., and R. Werlang, 1992. Uncertainty Aversion, Risk Aversion, and the Optimal Choice of Portfolio, Econometrica 60, 197-204.

Dumas, B., 1989. Two-Person Dynamic Equilibrium in Capital Markets, Review of Financial Studies, 2, 157-188.

Ellsberg, D., 1961. Risk, Ambiguity and the Savage Axioms, Quarterly Journal of Economics, 25, 643-669

Epstein, L. and J. Miao, 2003. A two-Person Dynamic Equilibrium under Ambiguity, Journal of Economic Dynamics and Control, 27, 1253-1288.

Epstein, L., and M. Schneider, 2003. Recursive Multiple Priors, Journal of Economic Theory, 113, 32-50.

Epstein, L. and T. Wang, 1994. Intertemporal Asset Pricing under Knightian Uncertainty, Econometrica, 62, 283-322.

Gagliardini, P., P. Porchia and F. Trojani, 2004. Ambiguity Aversion, Bond Pricing and the NonRobustness of some Affine Term Structures, Working Paper, University of Lugano, Switzerland.

Gagliardini P., G. Urga and F. Trojani, 2004. Robust GMM Tests for Structural Breaks, Journal of Econometrics, forthcoming. 
Ghirardato, P., F. Maccheroni and M. Marinacci, 2002. Ambiguity from the Differential Viewpoint, Discussion Paper, ICER

Gilboa, I., 1987. Expected Utility Theory with Purely Subjective Non-Additive Probabilities, Journal of Mathematical Economics, 16, 65-88.

Gilboa, I. and D. Schmeidler, 1989. Maxmin Expected Utility with Non-unique Prior, Journal of Mathematical Economics, 18, 141-153.

Hansen, L. P., T. J. Sargent, and D. Tallarini, Jr., 1999. Robust Permanent Income and Pricing, Review of Economic Studies, 66, 873-907.

Hansen, L.P., T. J. Sargent, G. A. Turmuhambetova, and N. Williams, 2001. Robustness and Uncertainty Aversion, Preprint University of Chicago.

Huber, P., 1981. Robust statistics, Wiley, New York.

Kim, T. S., and E. Omberg, 1996. Dynamic Nonmyopic Portfolio Behaviour, Review of Financial Studies, 9, 141-161.

Klibanoff, P., M. Marinacci and S. Mukerji, 2003. A Smooth Model of Decision Making under Ambiguity, Discussion Paper, ICER.

Kogan, L. and R. Uppal, 2000. Risk Aversion and Optimal Policies in Partial and General Equilibrium Economies, Working Paper, UBC.

Krishnakumar, J. and E. Ronchetti, 1997. Robust Estimators for Simultaneous Equations Models, Journal of Econometrics, 78, 295-314.

Lei, C. I., 2001. Why Don't Investors Have Large Positions in Stocks? A Robustness Perspective, Manuscript, Chicago University.

Liu, W., 2002. Saving and Portfolio Decision: Observable Implications from Knightian Uncertainty, Working Paper, University of Washington. 
Liu, J., J. Pan and T. Wang, 2002. An Equilibrium Model of Rare Events Premia, Manuscript.

Maenhout, P., 2001. Robust Portfolio Rules, Hedging and Asset Pricing, Working Paper, INSEAD.

Mancini, L., E. Ronchetti and F. Trojani, 2003. Optimal Conditionally Unbiased BoundedInfluence Inference in Dynamic Location and Scale Models, Working Paper, University of Lugano, Switzerland.

Ortelli, C. and F. Trojani, 2004. Robust Efficient Method of Moments, Journal of Econometrics, forthcoming.

Ronchetti, E. and F. Trojani, 2001. Robust Inference with GMM estimators, Journal of Econometrics, 101, 37-69.

Sakata, S. and H. White, 1998. High Breakdown Point Conditional Dispersion Estimation with Application to S\&P 500 Daily Returns Volatility, Econometrica 66, 529-567.

Sbuelz, A. and F. Trojani, 2002. Equilibrium Asset Pricing with Time Varying Pessimism, Working Paper, University of Lugano, Switzerland.

Tornell, A., 2000. Robust- $H_{\infty}$ Forecasting and Asset Pricing Anomalies, Working Paper, NBER Working Paper Series.

Trojani, F., and P. Vanini, 2001. Perturbative Solutions of Hamilton Jacobi Bellman Equations in Robust Decision Making, Working Paper, University of Lugano, Switzerland.

Trojani, F., and P. Vanini, 2002a. A Note on Robustness in Merton's Model of Intertemporal Consumption and Portfolio Choice, Journal of Economic Dynamics and Control, 26/3, p. 423435.

Trojani, F. and P. Vanini, 2002b. A Review of Perturbative Approaches for Robust Optimal Portfolio Problems. Computational Methods in Decision-Making, Economics and Finance', Kluwer Applied Optimization Series. 
Uppal, R., and T. Wang 2003. Model Misspecification and Under-Diversification, Journal of Finance, 58 (6), 2465-2486. 

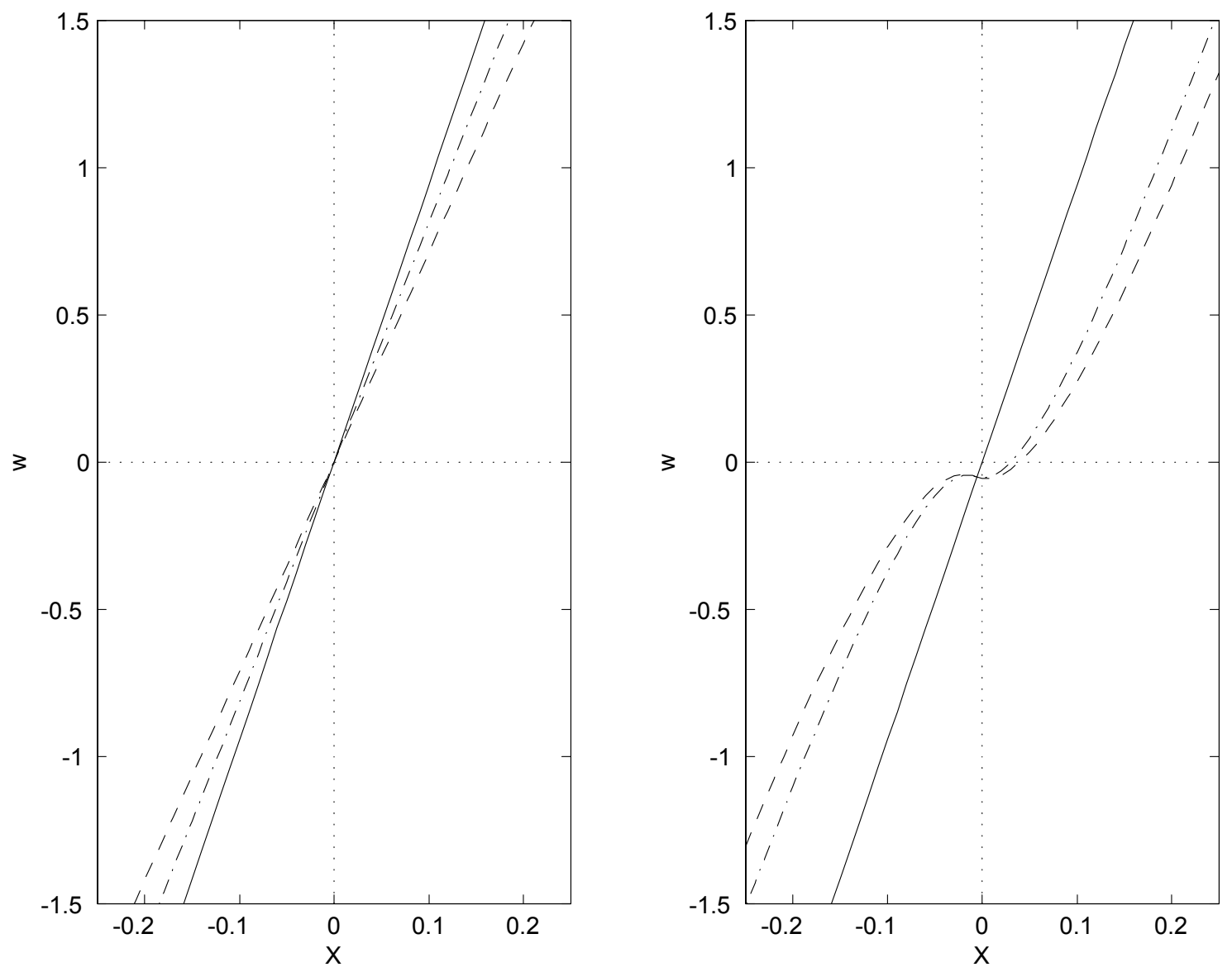

Figure 1: Optimal risky allocation $w$ as a function of the state $X$ for the parameter choices $r=0.05, \delta=0.06, \sigma=0.15, \xi=0.037, \lambda=0.0423, \bar{X}=0.0942, \gamma=0.25, \rho=0.90$. In the left panel we plot the portfolio policies in model (P1) for $\vartheta=0,0.1,0.2$ (straight, dash-dotted and dash lines). The right panel gives the portfolio policies in model (P2) for $\eta=0,0.005,0.01$ (straight, dash-dotted and dash curves). 


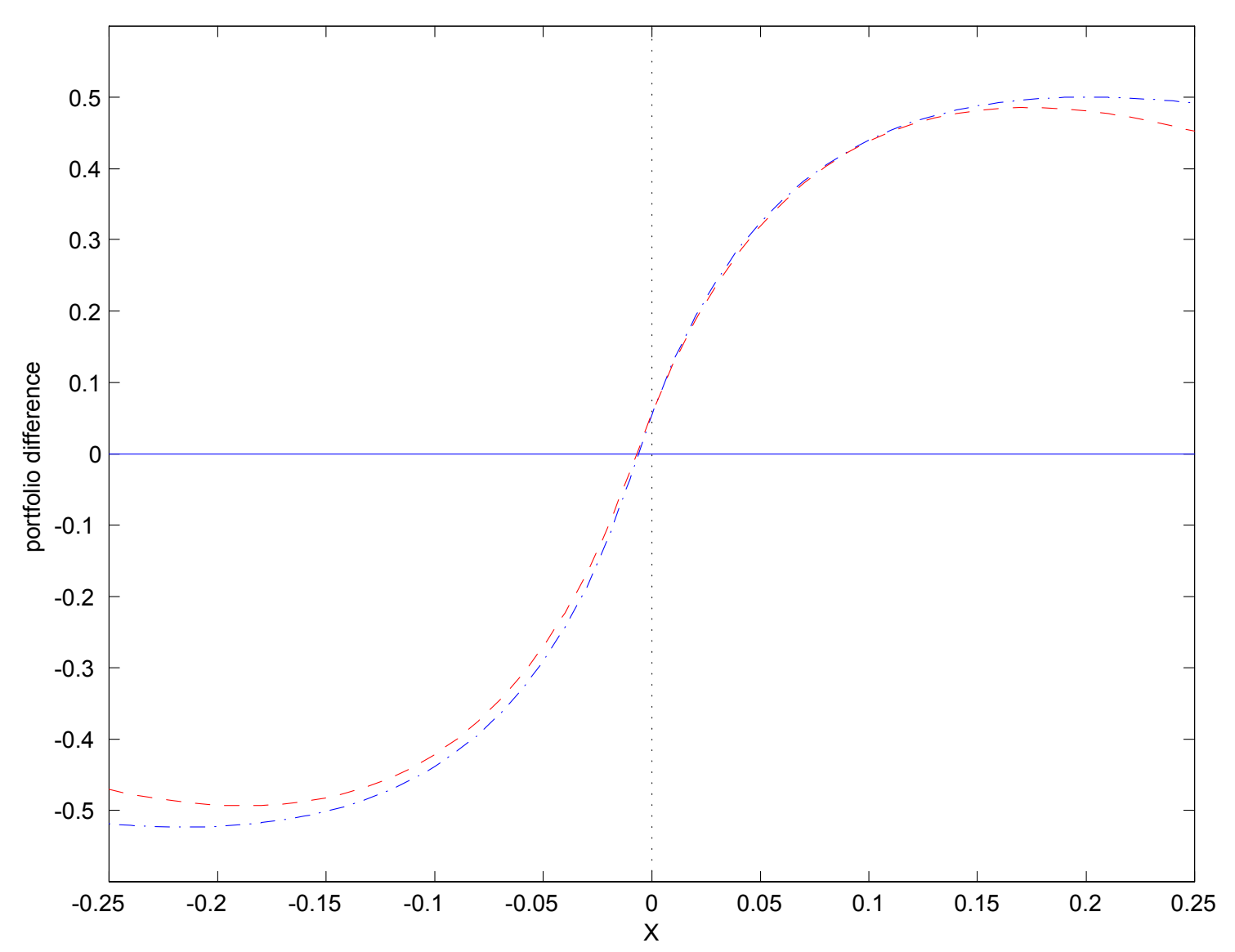

Figure 2: Differences in optimal risky allocations as a function of the state $X$ for the parameter choices $r=0.05, \delta=0.06, \sigma=0.15, \xi=0.037, \lambda=0.0423, \bar{X}=0.0942, \gamma=0.25, \rho=0.90$. We plot the difference of the portfolio policies in model (P1) for $\vartheta=0.1,0.2$ and the portfolio policies in model (P2) for $\eta=0.005,0.001$ ( dash-dotted and dash lines). 

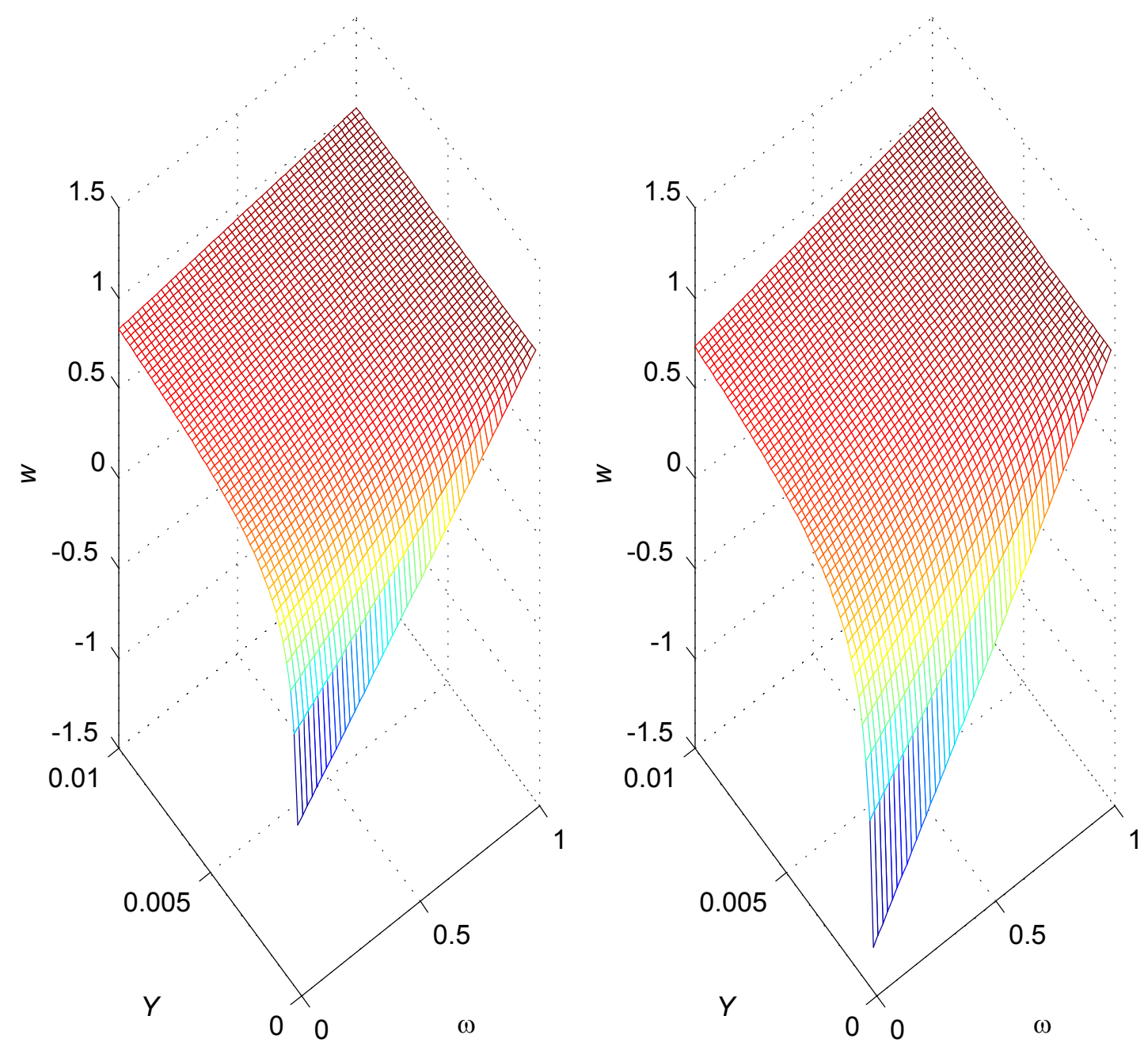

Figure 3: Optimal risky allocation $w$ under ambiguity as a function of the state $(\omega, Y)$ for the parameter choices $\delta=0.02, \sigma_{e}=0.02, \sigma_{Y}=0.01, \delta_{e Y}=-0.05, \gamma=0.25$. The plot in the left panel is for $\eta=0.001$. The one in the right panel is for $\eta=0.002$. 